\title{
The genus Eriastichus La Salle (Hymenoptera, Eulophidae, Tetrastichinae) in the Neotropical region, introducing 48 new species
}

\author{
Christer Hansson ${ }^{1,2}$ \\ I Scientific Associate Biological Museum (Entomology), Lund University, Sölvegatan 37, SE-22362 Lund, \\ Sweden 2 Natural History Museum, Life Sciences, Cromwell Road, London, UK \\ Corresponding author: Christer Hansson (christer.hansson@biol.lu.se) \\ Academic editor: N. Johnson | Received 4 November 2020 | Accepted 14 December 2020 | Published 22 February 2021 \\ http://zoobank.org/EE1BAF87-5BD7-4E18-9DF9-29E2C9CC7DAC \\ Citation: Hansson C (2021) The genus Eriastichus La Salle (Hymenoptera, Eulophidae, Tetrastichinae) in the \\ Neotropical region, introducing 48 new species. ZooKeys 1019: 35-91. https://doi.org/10.3897/zookeys.1019.60364
}

\begin{abstract}
Our knowledge of the recently described genus Eriastichus La Salle is greatly enhanced with the addition of 48 new species: E. acribis sp. nov., E. aphritis sp. nov., E. cluridis sp. nov., E. coelotis sp. nov., E. colenis sp. nov., E. copalensis sp. nov., E. daptilis sp. nov., E. decoris sp. nov., E. denotatis sp. nov., E. derilis sp. nov., E. diadrys sp. nov., E. dotaensis sp. nov., E. drupis sp. nov., E. ebulis sp. nov., E. egrestis sp. nov., E. eleagnis sp. nov., E. ellipsis sp. nov., E. eminis sp. nov., E. facilis sp. nov., E. fenestris sp. nov., E. follis sp. nov., E. galeatis sp. nov., E. geratis sp. nov., E. glanis sp. nov., E. hilaris sp. nov., E. johnlasallei sp. nov., E. johnnoyesi sp. nov., E. maniatis sp. nov., E. nebulis sp. nov., E. neonis sp. nov., E. nexilis sp. nov., E. novalis sp. nov., E. nugalis sp. nov., E. oasis sp. nov., E. ononis sp. nov., E. orestis sp. nov., E. pallidops sp. nov., E. parabilis sp. nov., E. renodis sp. nov., E. rivalis sp. nov., E. sannionis sp. nov., E. scalaris sp. nov., E. sodalis sp. nov., E. taraxis sp. nov., E. tendrilis sp. nov., E. tonioazofeifai sp. nov., E. velaminis sp. nov., and $E$. vestis sp. nov. All species are known only from males, and all material is from Costa Rica. Females show little morphological variation and are not possible to separate to species or link to conspecific males at present. Apart from the diagnostic features for the genus presented in the original description, two new autapomorphies are introduced here: an inflated pleural membrane between $\mathrm{Gt}_{1-4}$ and $\mathrm{Gs}_{1-4}$, and tufts of pale and flattened setae laterally on $\mathrm{Gt}_{4-6}$. Both features are found on the gaster and are present in both sexes. The distribution of the genus is firmly established as predominantly neotropical. The biology remains unknown.
\end{abstract}

\section{Keywords}

Autapomorphies, Costa Rica, identification key

Copyright Christer Hansson. This is an open access article distributed under the terms of the Creative Commons Attribution License (CC BY 4.0), which permits unrestricted use, distribution, and reproduction in any medium, provided the original author and source are credited. 


\section{Introduction}

Taxonomic knowledge of the Tetrastichinae (Chalcidoidea: Eulophidae) fauna in the Neotropical region is very poor, at both the genus and species levels. LaSalle et al. (2006) listed 29 genera in the subfamily from the Neotropical region, and with the addition of a new genus by Hansson (2020) this number is now 30, but hardly any of them have been investigated in this part of the world.

Eriastichus is a genus confined to the Americas, with the great majority of species occurring in tropical parts (La Salle 1994 and present findings). It was described in a paper on the Nearctic genera of Tetrastichinae (LaSalle 1994), including three species that were described in same paper. The species were based on females, and the male was included for only one of the species. With the exception of one specimen from the U.S.A. (Texas), the specimens were from the Neotropical region, ranging from the Caribbean (Dominican Republic) through Central America (Costa Rica, Mexico) to South America (Ecuador). The biology of the species was cited as being unknown.

\section{Materials and methods}

\section{Material}

Material forming the base of this study includes 63 females and 81 males, and all specimens were collected with a sweep net. The specimens were killed and kept in $80 \%$ ethanol until dried with a critical point drier. Thus, the specimens did not shrivel when dried and this should be kept in mind for the measurements/ratios given.

The types are deposited in the following museums with registration numbers prefixed by their acronyms:

MZLU Biological Museum (Entomology), Lund University, Lund, Sweden;

MZUCR Museo de Zoología, Universidad de Costa Rica, San José, Costa Rica;

NHMUK Natural History Museum, London, United Kingdom.

\section{Other museum acronyms}

CNC Canadian National Collection of Insects, Ottawa, Canada;

USNM United States National Museum of Natural History, Washington, D.C., USA.

\section{Imaging}

The colour images of the specimens were made using Canon camera equipment including an EOS 5D Mark IV body, a telezoom lens, 70-300 mm (but using only 135 $\& 200 \mathrm{~mm}$ ), with a $10 \times$ Mitutoyo microscope lens attached, and macro twin lite MT-24 EX for illumination. The camera was attached to a Cognisys stackshot macro- 
rail system. The picture stacking was done with Helicon Focus version 6, and Adobe Photoshop was used for image processing. The SEM micrographs are from uncoated specimens and were done with a Hitachi SU 3500 microscope, in low vacuum and using a backscatter detector.

\section{Morphological terminology}

The terminology used here follows Gibson (1997), except the term mesoscutellum which is used instead of scutellum.

\section{Abbreviations of morphological terms}

CC costal cell in fore wing;

F1-4 flagellomeres 1-4;

$\mathrm{Gs}_{1-7}$ gastral sternites 1-7;

$\mathbf{G t}_{1-7}$ gastral tergites 1-7;

HE height of eye;

MS malar space;

MV marginal vein;

OOL shortest distance between lateral ST stigmal vein in fore wing; ocelli and eyes;
PM postmarginal vein in fore wing;

POL shortest distance between lateral ocelli;

SLG sublateral groove on mesoscutellum (sublateral line in LaSalle 1994);

SMG submedian groove on mesoscutellum (submedian line in LaSalle 1994);

WM width of mouth opening.

\section{Species names}

To avoid the problems associated with the requirement by the International Code of Zoological Nomenclature (International Commission of Zoological Nomenclature 1999) that the gender of species names must agree with that of the genus, all new names proposed in the present study, except when indicated otherwise, must be treated as arbitrary combinations of letters or nouns in apposition. As a consequence, species names will not change if species are transferred to a genus with a different gender at any time in the future.

\section{Taxonomic accounts}

\section{Genus Eriastichus La Salle}

La Salle 1994: 173-174. Type species Eriastichus cigdemae La Salle.

Diagnosis. Heavily setose species (Figs 1, 2, 5, 6): head (including eyes), pronotum, mesoscutum, coxae, and lateral parts of propodeum (excl. bare median part - hairy only in E. masneri La Salle) \pm covered with fine, short setae; malar sulcus curved; base of scape oblique (e.g., Fig. 45); F1-F4 of male antenna with scattered setae of varying length 

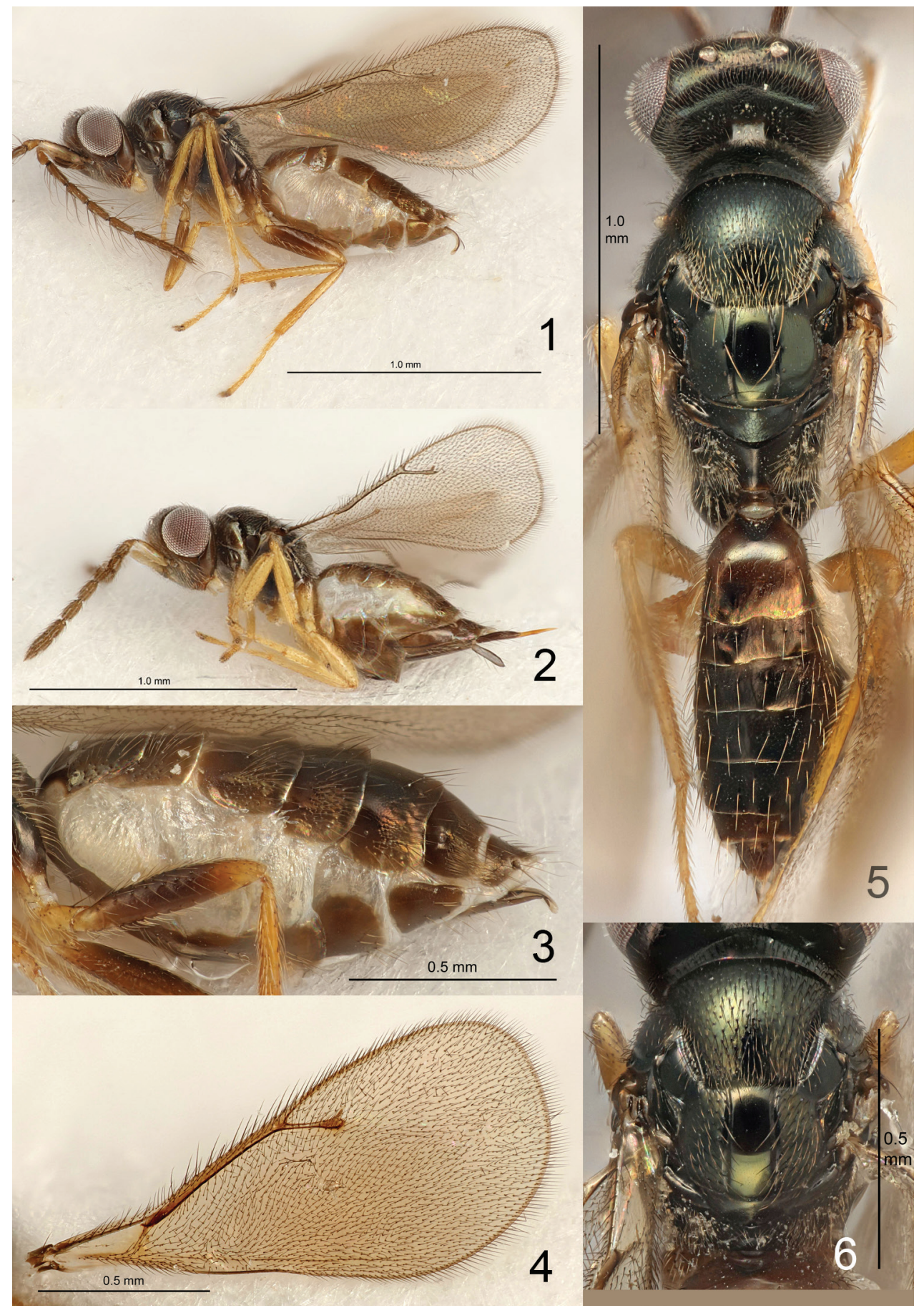

Figures I-6. Eriastichus spp. I E. eleagnis, body in lateral view, male paratype $\mathbf{2}$ E. sp., body in lateral view, female $3 E$. decoris, gaster in lateral view, male holotype $4 E$. sp., right fore wing, female $\mathbf{5}$ E. fenestris, body in dorsal view, male holotype 6 E. sp., thoracic dorsum, female. 


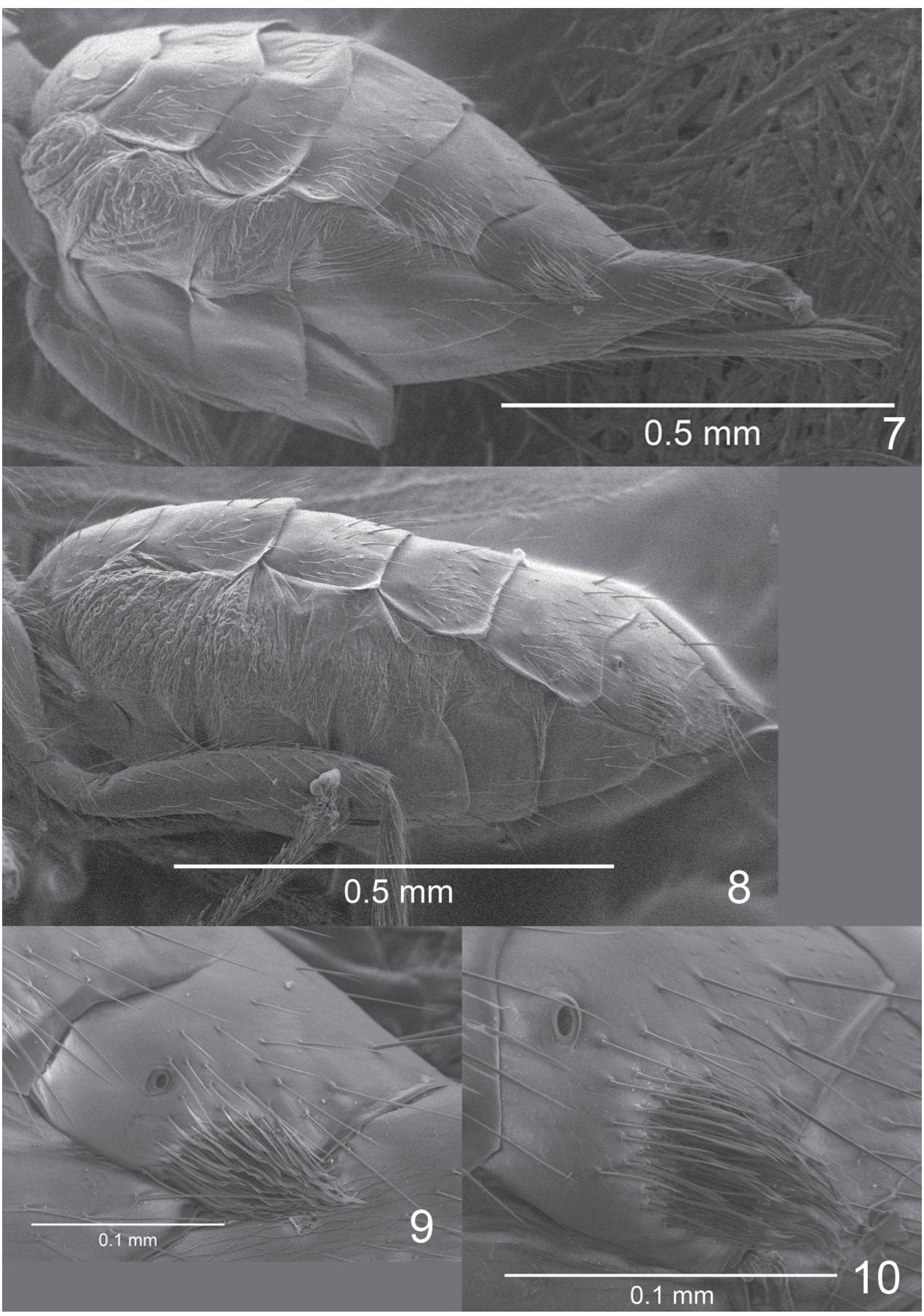

Figures 7-10. Eriastichus spp. 7, 8 gaster in lateral view: 7 E. sp., female 8 E. ebulis, male paratype 9, $10 \mathrm{Gt}_{6}$ in lateral view, with brush of flattened setae: $9 \mathrm{E}$. sp., female 10 E. ebulis, male paratype. 
(Figs 11-58), i.e., without a compact whorl of long setae attached close to base - a feature present in many tetrastichine groups; mesoscutellum transverse (Figs 5-6); dorsellum large, flat to slightly convex and smooth (Fig. 5); fore wing with postmarginal vein 0.3$0.7 \times$ as long as the elongate and slender stigmal vein, wing disc strongly setose, including base (i.e., without speculum) and ventral surface of costal cell (Fig. 4), wings \pm uniformly but weakly infuscate (Fig. 4) but in a few species hyaline; gaster with pleural membrane between $\mathrm{Gt}_{1-4}$ and $\mathrm{Gs}_{1-4}$ inflated (Figs 1-3, 7, 8); both sexes with a tuft of pale and flattened setae laterally on $\mathrm{Gt}_{3-6}$ (Figs 7-10), though most species with tufts only on $\mathrm{Gt}_{6}$.

\section{Key to males}

1 Mesoscutellum with parts lateral to submedian grooves strongly setose (Fig. 6) .................................................................................2

- Mesoscutellum with parts lateral to submedian grooves with two or three pairs of strong setae (Fig. 5)

Median propodeum hairy (La Salle 1994: fig. 30)

E. masneri La Salle (male unknown, this is an anticipated placement; not recorded from Costa Rica) Median propodeum bare (as in Fig. 5)

E. nakos La Salle (male unknown, this is an anticipated placement; not recorded from Costa Rica) Scape 1.3-1.4 $\times$ as long as ventral plaque (Fig. 50); lower face yellowish brown (Fig. 59)

E. pallidops sp. nov. Scape $1.7-8.0 \times$ as long as ventral plaque; colour of lower face variable ......4 Scape 5.3-8.0 $\times$ as long as ventral plaque. Scape $1.7-5.0 \times$ as long as ventral plaque Antenna with dorsobasal setae on F1 $0.5 \times$ as long as length of F1 (Fig. 15), pedicel + flagellum $2.0 \times$ as long as width of head...... E. copalensis sp. nov. Antenna with dorsobasal setae on F1 at least $1.1 \times$ as long as length of F1, pedicel + flagellum at most $1.6 \times$ as long as width of head Scape $8.0 \times$ as long as ventral plaque (Fig. 57), and $1.4 \times$ as long as height of eye

E. tonioazofeifai sp. nov.

Scape at most $6.7 \times$ as long as ventral plaque, and at most $0.9 \times$ as long as height of eye

Antennal flagellum longer (Fig. 11), e.g., F1 $2.5 \times$, F4 $2.8 \times$, clava $7.3 \times$ as long as wide

E. acribis sp. nov.

Antennal flagellum shorter, F1 1.5-1.7 ×, F4 $2.0 \times$, clava 4.8-5.2 $\times$ as long as wide

Setae on flagellomeres more erect, and dorsobasal setae on F1 $1.5 \times$ as long as length of F1 (Fig. 12). Setae on flagellomeres less erect, and dorsobasal setae on F1 $1.1 \times$ as long as length of F1 (Fig. 16) E. coelotis sp. nov. 
11 Dorsobasal setae on F1 $0.8-1.1 \times$ as long as length of F1 .........................12

12 Ventral plaque on scape situated medially (Fig. 37) ....E. johnlasallei sp. nov.

13 Each flagellomere with few setae, e.g., F1 with six setae, ventral plaque $0.2 \times$ as long as length of scape (Fig. 17)

E. daptilis sp. nov.

Each flagellomere with more setae, e.g., F1 with ca. 15 setae, ventral plaque $0.4 \times$ as long as length of scape (Fig. 20).....

E. dotaensis sp. nov. Flagellomeres longer, F1, F3, F4 each $3.7 \times$, F2 $4.0 \times$ as long as wide (Fig. 18) E. decoris sp. nov. Flagellomeres shorter, F1 2.0-3.5, F2 2.4-3.5, F3 2.1-3.4, F4 2.3-3.4 x as long as wide

15 Fore and mid legs with coxae and femora yellowish brown

E. denotatis sp. nov.

Fore and mid legs with coxae and femora dark brown. .16

16 Ventral plaque $0.5 \times$ as long as length of scape (Fig. 22).. E. diadrys sp. nov. Ventral plaque $0.3 \times$ as long as length of scape (Fig. 23).....E. ebulis sp. nov.

17 Dorsobasal setae on F1 ca. $2 \times$ as long as F1 E. cigdemae La Salle Dorsobasal setae on F1 at most $1.5 \times$ as long as F1 18

18 Dorsobasal setae on F1 at least $1.2 \times$ as long as F1, and setae on flagellomeres erect (Figs 24, 27)

Dorsobasal setae on F1 at most $1.0 \times$ as long as F1, setae on flagellomeres variable

Antenna shorter (Fig. 24), pedicel + flagellum $1.1 \times$ as long as width of head E. egrestis sp. nov. Antenna longer (Fig. 27), pedicel + flagellum 1.6-1.7 $\times$ as long as width of head E. eleagnis sp. nov. Scape 4.5-5.0 $\times$ as long as ventral plaque.................................................21 Scape $1.8-4.2 \times$ as long as ventral plaque 24 Ventral plaque situated medially (Fig. 25) E. ellipsis sp. nov. Ventral plaque situated below median part of scape 22 Dorsobasal setae on F1 $0.6 \times$ as long as length of F1 (Fig. 40)

E. johnnoyesi sp. nov.

Dorsobasal setae on F1 0.9-1.0 $\times$ as long as length of F1 (Figs 28, 31) Antenna with F1-F4 each 3.3-3.5 $\times$ as long as wide, setae on flagellomeres less erect (Fig. 31) E. eminis sp. nov. Antenna with F1-F4 1.8-2.5 $\times$ as long as wide, setae on flagellomeres more erect (Fig. 28) E. facilis sp. nov. Scape $1.7-2.0 \times$ as long as ventral plaque 
Setae on flagellomeres less erect, scape yellowish brown to pale brown ......26 Antenna with F1-F4 each $2.0 \times$ and clava $4.3 \times$ as long as wide (Fig. 21).....

E. derilis sp. nov.

Antenna with F1-F4 each 2.7-3.0 $\times$ and clava 5.5-6.3 $\times$ as long as wide (Figs 13, 30)

27 Flagellomeres with more setae, e.g., F2 with eight setae on dorsal surface (Fig. 13)

Flagellomeres with less setae, e.g., F2 with four setae on dorsal surface (Fig. 30) E. follis sp. nov.

Frons and legs yellowish brown

E. sannionis sp. nov.

Frons partly to completely dark brown, legs yellowish brown to dark brown

Coxae and femora \pm yellowish brown, some coxae or hind femur can be partly dark brown

Setae on flagellomeres erect (Figs 34, 58)

Setae on flagellomeres less erect than in alternate (Figs 33, 56)

Scape narrow at base (Fig. 34)

E. galeatis sp. nov. Scape wide at base (Fig. 58) E. vestis sp. nov.

32 Flagellomeres with few setae, e.g., F2 with three setae on dorsal surface (Fig. 56) ..................................................................... velaminis sp. nov. Flagellomeres with more setae, e.g., F2 with eight setae on dorsal surface (Fig. 33) E. geratis sp. nov.

Scape $3.5-4.2 \times$ as long as ventral plaque 34

Scape $2.2-3.1 \times$ as long as ventral plaque 37

Dorsobasal setae on F1 $0.5-0.6 \times$ as long as F1 (Figs 32, 36) 35

Dorsobasal setae on F1 $0.8-1.0 \times$ as long as F1 (Figs 39, 41) 36

Flagellomeres with more setae, e.g., F2 with eight setae on dorsal surface (Fig. 32) E. glanis sp. nov. Flagellomeres with fewer setae, e.g., F2 with five setae on dorsal surface (Fig. 36) E. maniatis sp. nov. Setae on flagellomeres more erect (Fig. 39) E. neonis sp. nov. Setae on flagellomeres less erect (Fig. 41) E. nexilis sp. nov. Ventral plaque situated below middle of scape. 38 Ventral plaque situated in the middle, or slightly above the middle............44 Scape $2.2-2.5 \times$ as long as ventral plaque. 39 Scape $2.8-3.1 \times$ as long as ventral plaque 40 Dorsobasal setae on F1 $1.0 \times$ as long as F1 (Fig. 44) ........E. novalis sp. nov. Dorsobasal setae on F1 $0.5-0.6 \times$ as long as F1 (Fig. 42) .....E. oasis sp. nov. 
41 Flagellomeres longer, e.g., F2 3.6-3.8 $\times$ as long as wide (Figs 38, 43) .....42

- $\quad$ Flagellomeres shorter, F2 3.0-3.2 $\times$ as long as wide ..............................43

42 Dorsobasal setae on F1 $0.9 \times$ as long as F1 (Fig. 43) .........E. ononis sp. nov.

- $\quad$ Dorsobasal setae on F1 $0.6 \times$ as long as F1 (Fig. 38) ........E. nebulis sp. nov.

43 Dorsobasal setae on F1 $0.5 \times$ as long as F1, scape dark brown (Fig. 46)

E. parabilis sp. nov.

- $\quad$ Dorsobasal setae on F1 $0.9 \times$ as long as F1, scape yellowish brown (Fig. 49)....

E. orestis sp. nov.

44 Dorsobasal setae on F1 $0.5 \times$ as long as F1 and pedicel + flagellum $1.9 \times$ as long as width of head....

45

- $\quad$ Dorsobasal setae on F1 at least $0.6 \times$ as long as F1 and/or pedicel + flagellum at most $1.7 \times$ as long as width of head 46

45 Setae on flagellomeres more erect (Fig. 47) E. rivalis sp. nov. Setae on flagellomeres less erect (Fig. 45) E. nugalis sp. nov.

46 Dorsobasal setae on F1 $0.4 \times$ as long as F1 (Fig. 35) and pedicel + flagellum $1.7 \times$ as long as width of head E. hilaris sp. nov.

- Dorsobasal setae on F1 $0.6-1.0 \times$ as long as F1, if dorsobasal setae on F1 is only $0.6 \times$ as long as F1, then pedicel + flagellum are $1.5 \times$ as long as width of head

Antennal flagellum long and slender (Fig. 54), e.g., F4 $3.4 \times$ and clava $7.6 \times$ as long as wide

E. renodis sp. nov.

- $\quad$ F4 2.2-2.8 $\times$ and clava 4.8-6.3 $\times$ as long as wide 48

48 Dorsobasal setae on F1-F4 $1.0 \times$ as long as funicular segment it is attached to (Fig. 52)

E. scalaris sp. nov.

- $\quad$ Dorsobasal setae on F1-F4 $0.6-0.8 \times$ as long as funicular segment it is attached to

49 Antennal flagellum more slender (Fig. 53), e.g., F3 $3.0 \times$ and clava $6.3 \times$ as long as wide

E. taraxis sp. nov.

- $\quad$ F3 2.3-2.6 $\times$ and clava 4.8-5.8 $\times$ as long as wide

50

50 Pedicel + flagellum $1.9 \times$ as long as width of head

E. sodalis sp. nov.

Pedicel + flagellum 1.5-1.7 $\times$ as long as width of head

E. tendrilis sp. nov.

\section{Species treatments}

Eriastichus acribis sp. nov.

http://zoobank.org/4CAF1EDF-8C16-4075-B08B-1356397DEC4B

Figure 11

Type locality. Costa Rica, Heredia, Estación Biológica La Selva, 75 m, 10²6'N, $84^{\circ} 01^{\prime} \mathrm{W}, 24-25.1 i .2011$, J.S. Noyes leg.

Type specimen. Holotype male dried and glued to a paper card. Original labels: "COSTA RICA, Heredia, E.B. La Selva, 75 m, 10²6'N, 84º1'W, 24-25.ii.2011, 
J.S. Noyes, NHM (Ent) 2011-93", "HOLOTYPE Eriastichus acribis Hansson” [red printed label], (NHMUK014431021).

Diagnosis (male). Head brown; antenna (Fig. 11): scape yellowish brown, ventral plaque on scape ca. $0.1 \times$ as long as scape, antenna with dorsobasal setae on F1 $1.1 \times$ as long as F1; gaster with lateral tufts of pale and flattened setae on $\mathrm{Gt}_{6}$.

Description (male holotype NHMUK014431021). Length of body $1.2 \mathrm{~mm}$. Head brown. Antenna with scape yellowish brown, ventral plaque brown, pedicel and flagellum brown. Mesoscutum and mesoscutellum dark brown; dorsellum and propodeum pale brown. Legs yellowish brown. Gaster dark brown.

Head. Length/width in frontal view 0.8 ; width/length in dorsal view 2.5; POL/ OOL 2.8; WM/MS 3.6; MS/HE 0.4; HE/head length in frontal view 0.6; widths head/mesoscutum 1.2. Antenna. Pedicel + flagellum length/mesoscutum width 1.8; pedicel + flagellum length/head width 1.5 ; lengths scape/ventral plaque 6.7 ; ventral plaque located in the middle of scape; scape length/width 2.9; lengths scape/head (dorsal view) 0.5; scape length/HE 0.8; length/width F1, F2, F3, F4, clava: 2.5, 2.0, 2.3, 2.8, 7.3; length dorsobasal setae on F1/length F1 1.1. Mesosoma. Length/width 1.4; mesoscutum length/width 0.5 ; mesoscutellum length/width 0.6; widths SMG/SLG 1.0; enclosed space between SMG length/width 2.0; lengths mesoscutum/mesoscutellum 1.2; lengths mesoscutellum/dorsellum 2.3; lengths mesosoma/gaster 0.7. Wings. CC length/width 21.9; lengths CC/MV 1.0; lengths MV/ST 2.3; lengths MV/PM 4.9; lengths PM/ST 0.5; submarginal vein with four setae on dorsal surface. Gaster. With lateral tufts of pale and flattened setae on $\mathrm{Gt}_{6}$.

\section{Eriastichus aphritis sp. nov.}

http://zoobank.org/B8A47821-A1EE-4B64-B7C7-5EEE1B059956

Figure 13

Type locality. Costa Rica, Puntarenas, San Vito, Estación Biológica Las Alturas, $1500 \mathrm{~m}, 8^{\circ} 57^{\prime} \mathrm{N}, 82^{\circ} 50^{\prime} \mathrm{W}, 17-18 . i i .2012$, J.S. Noyes leg.

Type specimen. Holotype male dried and glued to a paper card. Original labels: "COSTA RICA, Puntarenas, San Vito, E.B. Las Alturas, $1500 \mathrm{~m}, 8^{\circ} 57^{\prime} \mathrm{N}, 82^{\circ} 50^{\prime} \mathrm{W}$, 17-18.ii.2012, J.S. Noyes, NHM (Ent) 2012-91", "HOLOTYPE Eriastichus aphritis Hansson" [red printed label], (NHMUK014431022).

Diagnosis (male). Head black with metallic tinges with part below antennal toruli yellowish brown, scape and pedicel pale brown, flagellum brown; ventral plaque on scape ca. $0.5 \times$ as long as scape (Fig. 13), antenna with dorsobasal setae on F1 $0.5 \times$ as long as F1; gaster with lateral tufts of pale and flattened setae on $\mathrm{Gt}_{6}$.

Description (male holotype NHMUK014431022). Length of body $2.2 \mathrm{~mm}$. Head black with metallic tinges with part below antennal toruli yellowish brown, scape and pedicel pale brown, flagellum brown, ventral plaque dark brown. Mesoscutum 


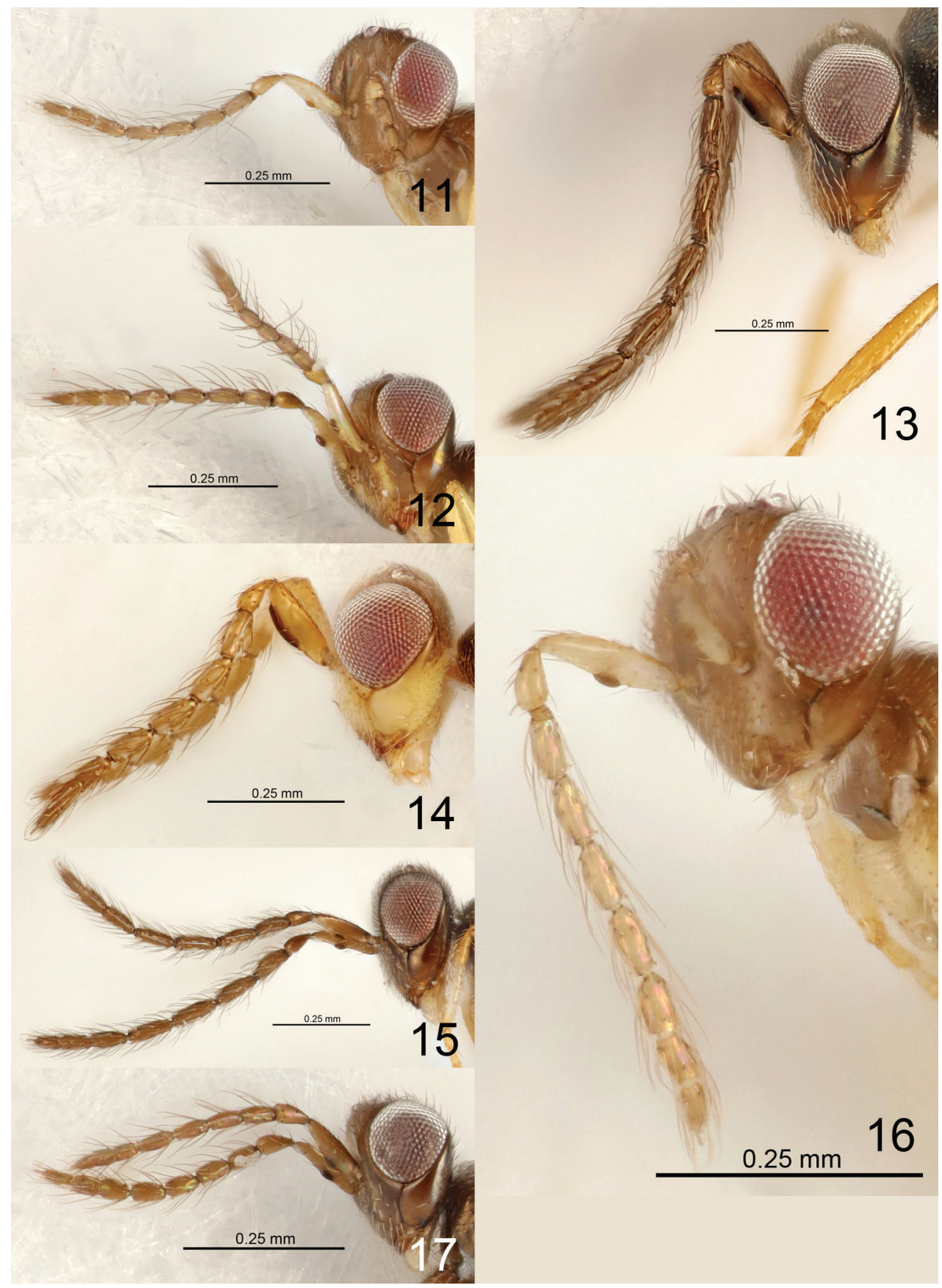

Figures I I-I 7. Eriastichus spp. head and antenna in lateral view, male holotypes I I E. acribis $\mathbf{I} 2$ E. cluridis $\mathbf{1 3}$ E. aphritis $\mathbf{1 4}$ E. colenis $\mathbf{1 5}$ E. copalensis $\mathbf{1 6}$ E. coelotis $\mathbf{1 7}$ E. daptilis. 
and mesoscutellum black with metallic tinges, dorsellum and propodeum dark brown. Legs yellowish brown. Gaster dark brown.

Head. Length/width in frontal view 0.8; width/length in dorsal view 2.5; POL/ OOL 1.9; WM/MS 1.5; MS/HE 0.6; HE/head length in frontal view 0.5; widths head/mesoscutum 1.2. Antenna. Pedicel + flagellum length/mesoscutum width 1.9; pedicel + flagellum length/head width 1.8 ; lengths scape/ventral plaque 2.1 ; ventral plaque located below the middle of scape; scape length/width 3.0; lengths scape/head (dorsal view) 0.5; scape length/HE 0.9; length/width F1, F2, F3, F4, clava: 3.0, 2.9, 2.8, 2.8, 5.5; length dorsobasal setae on F1/length F1 0.5. Mesosoma. Length/width 1.6; mesoscutum length/width 0.6; mesoscutellum length/width 0.8 ; widths SMG/ SLG 1.1; enclosed space between SMG length/width 2.3; lengths mesoscutum/mesoscutellum 1.5; lengths mesoscutellum/dorsellum 3.4; lengths mesosoma/gaster 0.7. Wings. CC length/width 17.5; lengths CC/MV 1.1; lengths MV/ST 2.1; lengths MV/PM 3.0; lengths PM/ST 0.7; submarginal vein with ten setae on dorsal surface. Gaster. With lateral tufts of pale and flattened setae on $\mathrm{Gt}_{6}$.

\section{Eriastichus cigdemae La Salle}

Eriastichus cigdemae La Salle, 1994: 207. Holotype female in USNM, not examined.

Diagnosis. Mesoscutellum with 2-3 pairs of setae; male with dorsobasal setae on F1 ca. $2 \times$ as long as F1.

Description. See La Salle (1994).

Distribution. Costa Rica, Mexico, U.S.A. (Texas) (La Salle 1994).

\section{Eriastichus cluridis sp. nov.}

http://zoobank.org/0C4B7F59-E6A9-4F47-AC10-BA87174C2738

Figure 12

Type locality. Costa Rica, Alajuela, Parque Nacional Volcan Arenal, $10^{\circ} 26^{\prime} \mathrm{N}, 84^{\circ} 43^{\prime} \mathrm{W}$, $600 \mathrm{~m}, 26$. ii.2013, J.S. Noyes leg.

Type specimen. Holotype male dried and glued to a paper card. Original labels: "COSTA RICA, Alajuela, P.N. Volcan Arenal, 10²6' N, 84²43'W, 600 m, 26.ii.2013, J.S. Noyes, NHM (Ent) 2013-145AQ", "HOLOTYPE Eriastichus cluridis Hansson" [red printed label],(NHMUK014431023).

Diagnosis (male). Head dark brown with part below antennal toruli yellowish brown; antenna (Fig. 12): scape yellowish brown, ventral plaque on scape ca. $0.2 \times$ as long as scape, antenna with dorsobasal setae on F1 $1.5 \times$ as long as F1; gaster with lateral tufts of pale and flattened setae on $\mathrm{Gt}_{6}$.

Description (male holotype NHMUK014431023). Length of body $1.2 \mathrm{~mm}$. Head dark brown with part below antennal toruli yellowish brown. Antenna with 
scape yellowish brown, ventral plaque dark brown, pedicel and flagellum brown. Mesoscutum, mesoscutellum and propodeum dark brown; dorsellum pale brown. Legs yellowish brown, fore and mid coxae brown at base, hind coxa and hind femur brown. Gaster dark brown.

Head. Length/width in frontal view 0.8; width/length in dorsal view 2.1; POL/ OOL 2.7; WM/MS 1.5; MS/HE 0.5; HE/head length in frontal view 0.6; widths head/mesoscutum 1.1. Antenna. Pedicel + flagellum length/mesoscutum width 1.6; pedicel + flagellum length/head width 1.4; lengths scape/ventral plaque 5.3; ventral plaque located in the middle of scape; scape length/width 3.0; lengths scape/head (dorsal view) 0.5; scape length/HE 0.9; length/width F1, F2, F3, F4, clava: 1.7, 1.7, 2.0, 2.0, 5.2; length dorsobasal setae on F1/length F1 1.5. Mesosoma. Length/width 1.4; mesoscutum length/width 0.6; mesoscutellum length/width 0.7; widths SMG/SLG 1.2; enclosed space between SMG length/width 1.7; lengths mesoscutum/mesoscutellum 1.4; lengths mesoscutellum/dorsellum 2.7; lengths mesosoma/gaster 0.8. Wings. CC length/width 25.0; lengths CC/MV 1.3; lengths MV/ST 2.0; lengths MV/PM 3.0; lengths PM/ST 0.7; submarginal vein with five setae on dorsal surface. Gaster. With lateral tufts of pale and flattened setae on $\mathrm{Gt}_{6}$.

\section{Eriastichus coelotis sp. nov.}

http://zoobank.org/D18D5E27-7C12-44CC-BBBB-0D18240246CB

Figure 16

Type locality. Costa Rica, Heredia, Estación Biológica La Selva, 75 m, 10²6'N, $84^{\circ} 01^{\prime} \mathrm{W}, 23-24.1 i .2005$, J.S. Noyes leg.

Type specimen. Holotype male dried and glued to a paper card. Original labels: "COSTA RICA, Heredia, E.B. La Selva, 75 m, $10^{\circ} 26^{\prime} \mathrm{N}, 84^{\circ} 01^{\prime} \mathrm{W}, 23-24$. ii.2005, J.S. Noyes", "HOLOTYPE Eriastichus coelotis Hansson" [red printed label], (NHMUK014431024).

Diagnosis (male). Head dark brown; antenna (Fig. 16): scape yellowish brown, ventral plaque on scape ca. $0.2 \times$ as long as scape, antenna with dorsobasal setae on F1 $1.1 \times$ as long as F1; gaster with lateral tufts of pale and flattened setae on $\mathrm{Gt}_{6}$.

Description (male holotype NHMUK014431024). Length $1.1 \mathrm{~mm}$. Head dark brown. Antenna with scape yellowish brown, ventral plaque dark brown, pedicel and flagellum pale brown. Mesoscutum, mesoscutellum and propodeum dark brown; dorsellum pale brown. Legs yellowish brown with base of hind coxa brown. Gaster dark brown.

Head. Length/width in frontal view 0.8 ; width/length in dorsal view 2.3; POL/ OOL 2.7; WM/MS 1.5; MS/HE 0.4; HE/head length in frontal view 0.7; widths head/mesoscutum 1.2. Antenna. Pedicel + flagellum length/mesoscutum width 1.7; pedicel + flagellum length/head width 1.5; lengths scape/ventral plaque 5.5; ventral plaque located in the middle of scape; scape length/width 3.4; lengths scape/head (dorsal view) 0.6; scape length/HE 0.8; length/width F1, F2, F3, F4, clava: 1.5, 1.8, 1.8, 
2.0, 4.8; length dorsobasal setae on F1/length F1 1.1. Mesosoma. Length/width 1.5; mesoscutum length/width 0.6; mesoscutellum length/width 0.6; widths SMG/SLG 1.1; enclosed space between SMG length/width 1.8; lengths mesoscutum/mesoscutellum 1.5; lengths mesoscutellum/dorsellum 2.3; lengths mesosoma/gaster 0.8. Wings. CC length/width 22.5; lengths CC/MV 1.2; lengths MV/ST 2.1; lengths MV/PM 5.0; lengths PM/ST 0.4; submarginal vein with five setae on dorsal surface. Gaster. With lateral tufts of pale and flattened setae on $\mathrm{Gt}_{6}$.

\section{Eriastichus colenis sp. nov.}

http://zoobank.org/2FA90FB6-94AF-4ABE-B176-ED2970A9177D

Figure 14

Type locality. Costa Rica, Cartago, Humo, El Copal, 947'N, $83^{\circ} 45^{\prime} \mathrm{W}, 1050$ 1250 m, 29.ii-6.iii.2008, C. Hansson leg.

Type specimen. Holotype male dried and glued to a paper card. Original labels: “COSTA RICA, Cartago, Humo, El Copal, 947'N, 834' W, 1050-1250 m, 29.ii6.iii.2008, C. Hansson", "HOLOTYPE Eriastichus colenis Hansson" [red printed label], (MZLU:7032.1).

Diagnosis (male). Head yellowish brown with vertex and upper $1 / 2$ of occiput dark brown; antenna (Fig. 14): scape yellowish brown, ventral plaque on scape ca. 0.4 $x$ as long as scape, antenna with dorsobasal setae on F1 $0.5 \times$ as long as F1; gaster with lateral tufts of pale and flattened setae on $\mathrm{Gt}_{5-6}$.

Description (male holotype MZLU:7032.1). Length of body $1.6 \mathrm{~mm}$. Head yellowish brown with vertex and upper $1 / 2$ of occiput dark brown. Antenna with scape yellowish brown, ventral plaque dark brown, pedicel and flagellum pale brown. Mesoscutum and propodeum dark brown; mesoscutellum dark brown with metallic tinges; dorsellum pale brown. Legs yellowish brown. Gaster dark brown.

Head. Length/width in frontal view 0.7 ; width/length in dorsal view 2.1; POL/ OOL 1.9; WM/MS 1.5; MS/HE 0.6; HE/head length in frontal view 0.6; widths head/mesoscutum 1.1. Antenna. Pedicel + flagellum length/mesoscutum width 1.7; pedicel + flagellum length/head width 1.5 ; lengths scape/ventral plaque 2.7 ; ventral plaque located above the middle of scape; scape length/width 4.2; lengths scape/head (dorsal view) 0.8; scape length/HE 1.3; length/width F1, F2, F3, F4, clava: 2.5, 2.3, 2.2, 2.2, 5.0; length dorsobasal setae on F1/length F1 0.5. Mesosoma. Length/width 1.5; mesoscutum length/width 0.5; mesoscutellum length/width 0.8; widths SMG/ SLG 1.3; enclosed space between SMG length/width 2.0; lengths mesoscutum/mesoscutellum 1.2; lengths mesoscutellum/dorsellum 3.3; lengths mesosoma/gaster 0.8. Wings. CC length/width 21.7; lengths CC/MV 1.1; lengths MV/ST 1.9; lengths MV/ PM 4.8; lengths PM/ST 0.4; submarginal vein with eight setae on dorsal surface. Gaster. With lateral tufts of pale and flattened setae on $\mathrm{Gt}_{5-6}$. 


\section{Eriastichus copalensis sp. nov.}

http://zoobank.org/55B02464-2EB7-45F9-8862-78EBF38D0631

Figure 15

Type locality. Costa Rica, Cartago, Humo, El Copal, 947'N, 834' W, 1050 1250 m, 29.ii-6.iii.2008, C. Hansson leg.

Type specimen. Holotype male dried and glued to a paper card. Original labels: "COSTA RICA, Cartago, Humo, El Copal, 9 $47^{\prime} \mathrm{N}, 83^{\circ} 45^{\prime} \mathrm{W}, 1050-1250$ m, 29.ii6.iii.2008, C. Hansson", "HOLOTYPE Eriastichus copalensis Hansson" [red printed label], (MZLU:7033.1)

Diagnosis (male). Head dark brown; antenna (Fig. 15): scape brown, ventral plaque on scape ca. $0.2 \times$ as long as scape, antenna with dorsobasal setae on F1 $0.5 \times$ as long as F1; gaster with lateral tufts of pale and flattened setae on $\mathrm{Gt}_{6}$.

Description (male holotype MZLU:7033.1). Length of body $1.5 \mathrm{~mm}$. Head dark brown. Antenna brown, ventral plaque dark brown. Mesoscutum, mesoscutellum and propodeum dark brown; dorsellum pale brown. Legs with coxae and femora dark brown, trochanters, tibiae and tarsi yellowish brown. Gaster dark brown.

Head. Length/width in frontal view 0.8; width/length in dorsal view 2.1; POL/ OOL 2.5; WM/MS 1.7; MS/HE 0.5; HE/head length in frontal view 0.6; widths head/mesoscutum 1.2. Antenna. Pedicel + flagellum length/mesoscutum width 2.2; pedicel + flagellum length/head width 2.0; lengths scape/ventral plaque 6.0; ventral plaque located slightly above the middle of scape; scape length/width 3.8; lengths scape/head (dorsal view) 0.5; scape length/HE 0.8; length/width F1, F2, F3, F4, clava: 3.0, 3.4, 3.8, 3.4, 8.0; length dorsobasal setae on F1/length F1 0.5. Mesosoma. Length/width 1.6; mesoscutum length/width 0.6 ; mesoscutellum length/width 0.8 ; widths SMG/SLG 1.2; enclosed space between SMG length/width 2.2; lengths mesoscutum/mesoscutellum 1.4; lengths mesoscutellum/dorsellum 3.0; lengths mesosoma/ gaster 0.8. Wings. CC length/width 24.0; lengths CC/MV 1.1; lengths MV/ST 1.7; lengths MV/PM 3.1; lengths PM/ST 0.5; submarginal vein with seven setae on dorsal surface. Gaster. With lateral tufts of pale and flattened setae on $\mathrm{Gt}_{6}$.

Etymology. Named after collecting locality.

\section{Eriastichus daptilis sp. nov.}

http://zoobank.org/C60FFDEF-AF6E-434F-AD51-8FA8464C025B

Figure 17

Type locality. Costa Rica, Heredia, Estación Biológica La Selva, 75 m, 10²6'N, $84^{\circ} 01^{\prime} \mathrm{W}, 28-29$. ii.2008, J.S. Noyes leg.

Type specimen. Holotype male dried and glued to a paper card. Original labels:

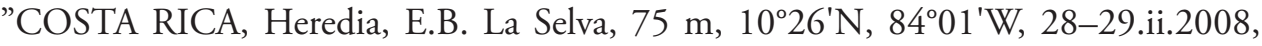


J.S. Noyes, NHM (Ent) 2010-21", "HOLOTYPE Eriastichus daptilis Hansson” [red printed label], (NHMUK014431025).

Diagnosis (male). Head and antenna dark brown; ventral plaque on scape ca. 0.2 $x$ as long as scape (Fig. 17), antenna with dorsobasal setae on F1 $1.1 \times$ as long as F1; dorsellum and major parts of legs dark brown; gaster without lateral tufts on tergites.

Description (male holotype NHMUK014431025). Length of body $0.9 \mathrm{~mm}$. Head and antenna dark brown, ventral plaque black. Mesoscutum, mesoscutellum, dorsellum and propodeum dark brown. Legs dark brown, trochanters and tarsi yellowish brown. Gaster dark brown.

Head. Length/width in frontal view 0.8 ; width/length in dorsal view 2.1; POL/ OOL 1.3; WM/MS 4.2; MS/HE 0.7; HE/head length in frontal view 0.6; widths head/mesoscutum 1.0. Antenna. Pedicel + flagellum length/mesoscutum width 1.6; pedicel + flagellum length/head width 1.5 ; lengths scape/ventral plaque 5.0; ventral plaque located below the middle of scape; scape length/width 3.6; lengths scape/head (dorsal view) 0.5; scape length/HE 0.9; length/width F1, F2, F3, F4, clava: 1.6, 1.8, 1.6, 1.6, 5.2; length dorsobasal setae on F1/length F1 1.1. Mesosoma. Length/width 1.3; mesoscutum length/width 0.5; mesoscutellum length/width 0.7; widths SMG/ SLG 1.1; enclosed space between SMG length/width 1.9; lengths mesoscutum/mesoscutellum 1.3; lengths mesoscutellum/dorsellum 2.5; lengths mesosoma/gaster 0.9. Wings. CC length/width 16.3; lengths CC/MV 1.2; lengths MV/ST 1.7; lengths MV/PM 2.8; lengths PM/ST 0.6; submarginal vein with four setae on dorsal surface.

Gaster. Without lateral tufts on tergites.

\section{Eriastichus decoris sp. nov.}

http://zoobank.org/A40C4518-95ED-4927-B6E7-5E3A49D80A33

Figures 3, 18

Type locality. Costa Rica, San José, San Gerardo de Dota, 9³3'N, 8347'W, 20-21. ii.2013, J.S. Noyes leg.

Type specimen. Holotype male dried and glued to a paper card. Original labels: "COSTA RICA, San José, San Gerardo de Dota, 20-21.ii.2013, J.S. Noyes, NHM (Ent) 2012-91", "HOLOTYPE Eriastichus decoris Hansson" [red printed label], (NHMUK014431026).

Diagnosis (male). Head black and antenna dark brown; ventral plaque on scape ca. $0.3 \times$ as long as scape (Fig. 18), antenna with dorsobasal setae on F1 $0.5 \times$ as long as F1; gaster with lateral tufts of pale and flattened setae on $\mathrm{Gt}_{5-6}$.

Description (male holotype NHMUK014431026). Length of body $2.3 \mathrm{~mm}$. Head black, antenna dark brown, ventral plaque black. Mesoscutum and mesoscutellum black with metallic tinges, dorsellum and propodeum black. Legs with coxae, hind femur and $4^{\text {th }}$ tarsomere on all legs dark brown, fore femur pale brown, trochanters, tibiae, mid femur, and tarsomeres 1-3 yellowish brown. Gaster dark brown.

Head. Length/width in frontal view 0.8 ; width/length in dorsal view 2.1 ; POL/ OOL 2.0; WM/MS 1.5; MS/HE 0.6; HE/head length in frontal view 0.5; widths 
head/mesoscutum 1.2. Antenna. Pedicel + flagellum length/mesoscutum width 2.1; pedicel + flagellum length/head width 2.1; lengths scape/ventral plaque 3.1; ventral plaque located below the middle of scape; scape length/width 3.4; lengths scape/head (dorsal view) 0.5; scape length/HE 0.9; length/width F1, F2, F3, F4, clava: 3.7, 4.0, 3.7, 3.7, 7.9; length dorsobasal setae on F1/length F1 0.5. Mesosoma. Length/width 1.6; mesoscutum length/width 0.7; mesoscutellum length/width 0.8 ; widths SMG/ SLG 1.1; enclosed space between SMG length/width 2.5; lengths mesoscutum/mesoscutellum 1.6; lengths mesoscutellum/dorsellum 2.6; lengths mesosoma/gaster 0.7. Wings. CC length/width 18.0; lengths CC/MV 0.9; lengths MV/ST 2.3; lengths MV/PM 3.8; lengths PM/ST 0.6; submarginal vein with nine setae on dorsal surface. Gaster. With lateral tufts of pale and flattened setae on $\mathrm{Gt}_{5-6}$.

\section{Eriastichus denotatis sp. nov.}

http://zoobank.org/306B5D9A-CBB9-447E-A4BD-57138BE907CD

Figures 19, 60

Type locality. Costa Rica, San José, San Gerardo de Dota, 9³3'N, 8347'W, 20-21. ii.2013, J.S. Noyes leg.

Type specimen. Holotype male dried and glued to a paper card. Original labels: "COSTA RICA, San José, San Gerardo de Dota, 20-21.ii.2013, J.S. Noyes, NHM (Ent) 2012-91", "HOLOTYPE Eriastichus denotatis Hansson" [red printed label], (NHMUK014431027).

Additional type material. Paratype $1 \delta^{\hat{~}}$ with same label data as holotype (NHMUK014431028).

Diagnosis (male). Head black (Fig. 60) and antenna dark brown (Fig. 19); ventral plaque on scape ca. $0.4 \times$ as long as scape (Fig. 19), antenna with dorsobasal setae on F1 $0.6 \times$ as long as F1; gaster with lateral tufts of pale and flattened setae on $\mathrm{Gt}_{6}$.

Description (male holotype NHMUK014431027). Length of body $2.6 \mathrm{~mm}$ (paratype $2.3 \mathrm{~mm}$ ). Head black, antenna dark brown, ventral plaque black. Mesoscutum and mesoscutellum black with metallic tinges, dorsellum and propodeum black. Legs with hind coxa dark brown, hind femur brown, fore and mid coxae and femora, trochanters, tibiae and tarsi yellowish brown. Gaster dark brown.

Head. Length/width in frontal view 0.8 ; width/length in dorsal view 2.1; POL/ OOL 1.6; WM/MS 1.5; MS/HE 0.6; HE/head length in frontal view 0.5; widths head/mesoscutum 1.1. Antenna. Pedicel + flagellum length/mesoscutum width 2.4; pedicel + flagellum length/head width 2.3; lengths scape/ventral plaque 2.4; ventral plaque located in the middle of scape; scape length/width 3.0; lengths scape/head (dorsal view) 0.5; scape length/HE 1.0; length/width F1, F2, F3, F4, clava: 3.1, 3.4, 3.4, 3.4, 7.3; length dorsobasal setae on F1/length F1 0.6. Mesosoma. Length/width 1.8; mesoscutum length/width 0.8; mesoscutellum length/width 0.9; widths SMG/SLG 1.2; enclosed space between SMG length/width 2.9; lengths mesoscutum/mesoscutellum 1.6; lengths mesoscutellum/dorsellum 2.7; lengths mesosoma/gaster 0.7. Wings. CC length/width 21.7; lengths CC/MV 0.9; lengths MV/ST 2.3; lengths MV/PM 


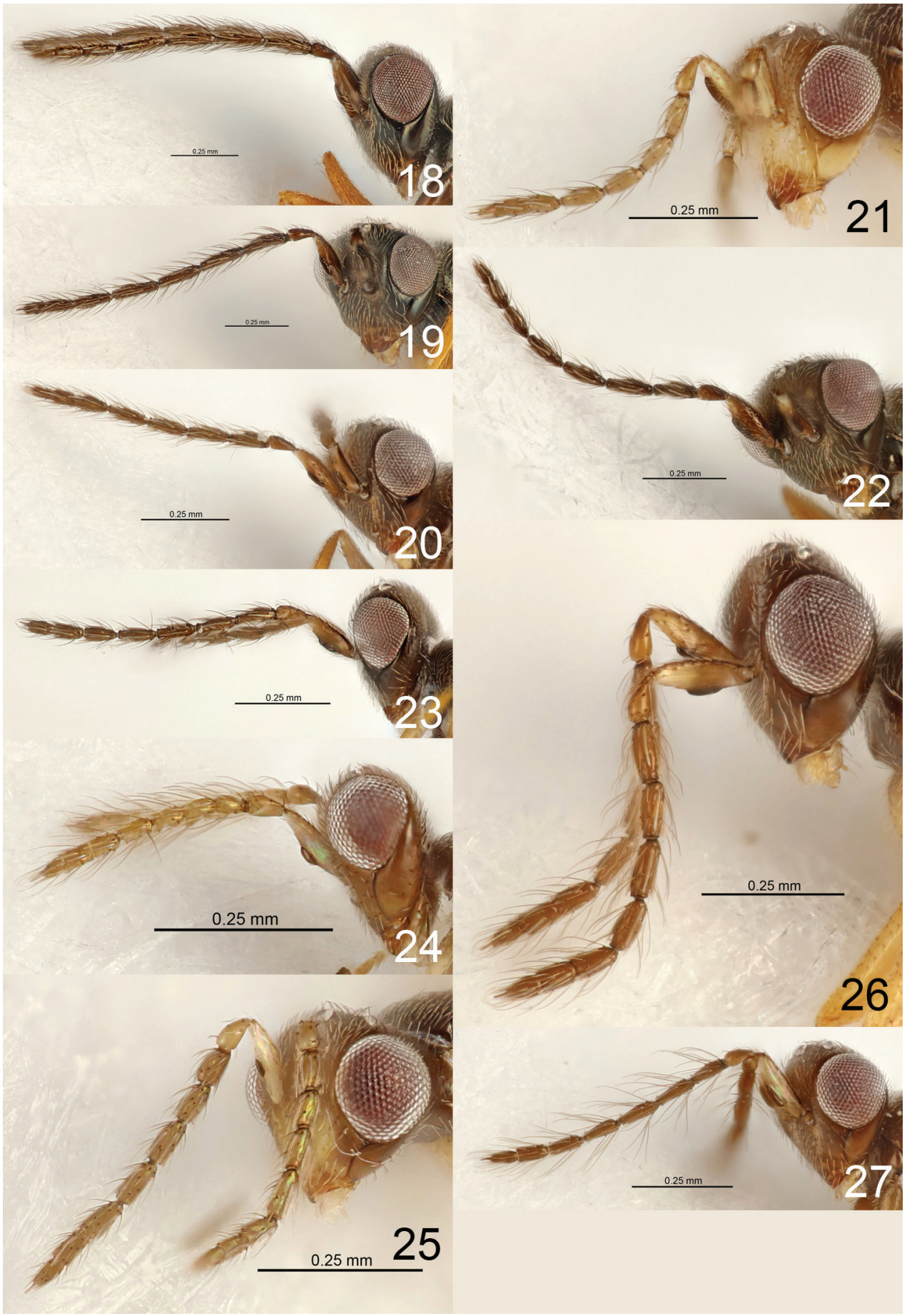

Figures 18-27. Eriastichus spp. head and antenna in lateral view, male holotypes $\mathbf{1 8}$ E. decoris $\mathbf{1 9}$ E. denotatis $\mathbf{2 0}$ E. dotaensis $\mathbf{2 1}$ E. derilis $\mathbf{2 2}$ E. diadrys $\mathbf{2 3}$ E. ebulis $\mathbf{2 4}$ E. egrestis $\mathbf{2 5}$ E. ellipsis $\mathbf{2 6}$ E. drupis 27 E. eleagnis. 
3.9; lengths PM/ST 0.6; submarginal vein with nine setae (ten setae in paratype) on dorsal surface. Gaster. With lateral tufts of pale and flattened setae on $\mathrm{Gt}_{6}$.

\section{Eriastichus derilis sp. nov.}

http://zoobank.org/59EEBC97-92F5-43EB-9205-3816D5D672B0

Figure 21

Type locality. Costa Rica, Cartago, Humo, El Copal, $9^{\circ} 47^{\prime} \mathrm{N}, 83^{\circ} 45^{\prime} \mathrm{W}, 1050$ 1250 m, 29.ii-6.iii.2008, C. Hansson leg.

Type specimen. Holotype male dried and glued to a paper card. Original labels: "COSTA RICA, Cartago, Humo, El Copal, 947'N, 8345'W, 1050-1250 m, 29.ii6.iii.2008, C. Hansson", "HOLOTYPE Eriastichus derilis Hansson" [red printed label], (MZLU:7034.1).

Diagnosis (male). Head with lower $1 / 2$ yellowish brown and upper $1 / 2$ dark brown, scape yellowish brown, pedicel and flagellum brown; ventral plaque on scape ca. 0.5 $x$ as long as scape (Fig. 21), antenna with dorsobasal setae on F1 $0.6 \times$ as long as F1; gaster with lateral tufts of pale and flattened setae on $\mathrm{Gt}_{5-6}$.

Description (male holotype MZLU:7034.1). Length of body $1.2 \mathrm{~mm}$. Head with lower $1 / 2$ yellowish brown and upper $1 / 2$ dark brown, scape yellowish brown, pedicel and flagellum brown, ventral plaque dark brown. Mesoscutum, mesoscutellum, dorsellum and propodeum dark brown. Legs yellowish brown. Gaster dark brown.

Head. Length/width in frontal view 0.8 ; width/length in dorsal view 2.3; POL/ OOL 1.9; WM/MS 1.6; MS/HE 0.5; HE/head length in frontal view 0.6; widths head/mesoscutum 1.2. Antenna. Pedicel + flagellum length/mesoscutum width 1.7; pedicel + flagellum length/head width 1.5 ; lengths scape/ventral plaque 2.0; ventral plaque located above the middle of scape; scape length/width 3.0; lengths scape/head (dorsal view) 0.5; scape length/HE 0.9; length/width F1, F2, F3, F4, clava: 2.0, 2.0, 2.0, 2.0, 4.3; length dorsobasal setae on F1/length F1 0.6. Mesosoma. Length/width 1.4; mesoscutum length/width 0.6; mesoscutellum length/width 0.9; widths SMG/ SLG 1.2; enclosed space between SMG length/width 2.2; lengths mesoscutum/mesoscutellum 1.3; lengths mesoscutellum/dorsellum 4.0; lengths mesosoma/gaster 0.8. Wings. CC length/width 21.0; lengths CC/MV 1.1; lengths MV/ST 1.8; lengths MV/ PM 5.0; lengths PM/ST 0.4; submarginal vein with six setae on dorsal surface. Gaster. With lateral tufts of pale and flattened setae on $\mathrm{Gt}_{5-6}$.

\section{Eriastichus diadrys sp. nov.}

http://zoobank.org/08F408C7-5C9C-4BFA-9A92-C11C2A11C881

Figure 22

Type locality. Costa Rica, San José, San Gerardo de Dota, 9³3'N, 8347'W, 20-21. ii.2013, J.S. Noyes leg. 
Type specimen. Holotype male dried and glued to a paper card. Original labels: "COSTA RICA, San José, San Gerardo de Dota, 20-21.ii.2013, J.S. Noyes, NHM (Ent) 2012-91", "HOLOTYPE Eriastichus diadrys Hansson" [red printed label], (NHMUK014431029).

Diagnosis (male). Head black and antenna dark brown; ventral plaque on scape ca. $0.5 \times$ as long as scape (Fig. 22), antenna with dorsobasal setae on F1 $0.5 \times$ as long as F1; gaster with lateral tufts of pale and flattened setae on $\mathrm{Gt}_{3-6}$.

Description (male holotype NHMUK014431029). Length of body $2.1 \mathrm{~mm}$. Head black, antenna dark brown, ventral plaque black. Mesoscutum and mesoscutellum black with metallic tinges, dorsellum and propodeum black. Legs with hind coxa and hind femur dark brown, fore and mid coxae yellowish brown with base dark brown, trochanters, tibiae and tarsi yellowish brown. Gaster dark brown.

Head. Length/width in frontal view 0.7 ; width/length in dorsal view 2.2; POL/ OOL 2.0; WM/MS 1.9; MS/HE 0.5; HE/head length in frontal view 0.6; widths head/mesoscutum 1.1. Antenna. Pedicel + flagellum length/mesoscutum width 1.9; pedicel + flagellum length/head width 1.8 ; lengths scape/ventral plaque 2.1 ; ventral plaque located in the middle of scape; scape length/width 2.7; lengths scape/head (dorsal view) 0.5; scape length/HE 0.9; length/width F1, F2, F3, F4, clava: 2.7, 2.9, 2.7, 2.6, 5.7; length dorsobasal setae on F1/length F1 0.5. Mesosoma. Length/width 1.6; mesoscutum length/width 0.7 ; mesoscutellum length/width 0.8 ; widths SMG/SLG 1.5; enclosed space between SMG length/width 2.0; lengths mesoscutum/mesoscutellum 1.6; lengths mesoscutellum/dorsellum 2.9; lengths mesosoma/gaster 0.8. Wings. CC length/width 20.6; lengths CC/MV 1.0; lengths MV/ST 2.1; lengths MV/PM 4.8; lengths PM/ST 0.4; submarginal vein with eight setae on dorsal surface. Gaster. With lateral tufts of pale and flattened setae on $\mathrm{Gt}_{3-6}$.

\section{Eriastichus dotaensis sp. nov.}

http://zoobank.org/E2228FB1-DE88-47A6-A504-CDEDE886BB50

Figure 20

Type locality. Costa Rica, San José, San Gerardo de Dota, 9³3'N, 834ㄱ'W, 20-21. ii.2013, J.S. Noyes leg.

Type specimen. Holotype male dried and glued to a paper card. Original labels: "COSTA RICA, San José, San Gerardo de Dota, 20-21.ii.2013, J.S. Noyes, NHM (Ent) 2012-91", "HOLOTYPE Eriastichus dotaensis Hansson" [red printed label], (NHMUK014431030).

Diagnosis (male). Head and antenna dark brown; ventral plaque on scape ca. 0.4 $x$ as long as scape (Fig. 20), antenna with dorsobasal setae on F1 $0.8 \times$ as long as F1; gaster with lateral tufts of pale and flattened setae on $\mathrm{Gt}_{6}$.

Description (male holotype NHMUK014431030). Length of body $1.6 \mathrm{~mm}$. Head and antenna dark brown, ventral plaque black. Mesoscutum and mesoscutellum black with metallic tinges, dorsellum pale brown, propodeum dark brown. Legs with 
coxae and femora dark brown, trochanters, tibiae and tarsi yellowish brown. Gaster dark brown.

Head. Length/width in frontal view 0.7; width/length in dorsal view 2.2; POL/ OOL 1.7; WM/MS 1.5; MS/HE 0.6; HE/head length in frontal view 0.6; widths head/mesoscutum 1.2. Antenna. Pedicel + flagellum length/mesoscutum width 2.1; pedicel + flagellum length/head width 1.9; lengths scape/ventral plaque 2.7 ; ventral plaque located below the middle of scape; scape length/width 3.0; lengths scape/head (dorsal view) 0.6; scape length/HE 1.0; length/width F1, F2, F3, F4, clava: 2.5, 2.8, 2.7, 2.7, 6.4; length dorsobasal setae on F1/length F1 0.8. Mesosoma. Length/width 1.5; mesoscutum length/width 0.7; mesoscutellum length/width 0.8 ; widths SMG/ SLG 1.1; enclosed space between SMG length/width 2.2; lengths mesoscutum/mesoscutellum 1.6; lengths mesoscutellum/dorsellum 2.6; lengths mesosoma/gaster 0.8 . Wings. CC length/width 26.0; lengths CC/MV 1.0; lengths MV/ST 2.2; lengths MV/ PM 3.6; lengths PM/ST 0.6; submarginal vein with six setae on dorsal surface. Gaster. With lateral tufts of pale and flattened setae on $\mathrm{Gt}_{6}$.

Etymology. Named after collecting locality.

\section{Eriastichus drupis sp. nov.}

http://zoobank.org/4CF0E18A-2BE0-4E3F-BB19-378178073525

Figure 26

Type locality. Costa Rica, Puntarenas, San Vito, Estación Biológica Las Alturas, $1500 \mathrm{~m}, 8^{\circ} 57^{\prime} \mathrm{N}, 82^{\circ} 50^{\prime} \mathrm{W}, 17-18 . i i .2012$, J.S. Noyes leg.

Type specimen. Holotype male dried and glued to a paper card. Original labels: "COSTA RICA, Puntarenas, San Vito, E.B. Las Alturas, 1500 m, 857'N, 82 50'W, 17-18.ii.2012, J.S. Noyes, NHM (Ent) 2012-91”, "HOLOTYPE Eriastichus drupis Hansson" [red printed label], (NHMUK014431031).

Diagnosis (male). Head dark brown, scape and pedicel pale brown, flagellum brown; ventral plaque on scape ca. $0.4 \times$ as long as scape (Fig. 26), antenna with dorsobasal setae on F1 $0.8 \times$ as long as F1; gaster with lateral tufts of pale and flattened setae on $\mathrm{Gt}_{5-6}$.

Description (male holotype NHMUK014431031). Length of body $1.4 \mathrm{~mm}$. Head dark brown, scape and pedicel pale brown, flagellum brown, ventral plaque dark brown. Mesoscutum and mesoscutellum black with metallic tinges, dorsellum pale brown, propodeum dark brown. Legs with hind coxa and hind femur dark brown, fore and mid coxae yellowish brown with base brown, remaining parts of legs yellowish brown. Gaster dark brown.

Head. Length/width in frontal view 0.8; width/length in dorsal view 2.2; POL/ OOL 1.8; WM/MS 1.6; MS/HE 0.5; HE/head length in frontal view 0.6; widths head/mesoscutum 1.3. Antenna. Pedicel + flagellum length/mesoscutum width 1.7; pedicel + flagellum length/head width 1.6; lengths scape/ventral plaque 2.8; ventral plaque located below the middle of scape; scape length/width 3.1 ; lengths scape/head 
(dorsal view) 0.5; scape length/HE 0.9; length/width F1, F2, F3, F4, clava: 2.7, 2.3, 2.3, 2.2, 5.3; length dorsobasal setae on F1/length F1 0.8. Mesosoma. Length/width 1.5; mesoscutum length/width 0.5; mesoscutellum length/width 0.7; widths SMG/ SLG 1.1; enclosed space between SMG length/width 2.1; lengths mesoscutum/mesoscutellum 1.3; lengths mesoscutellum/dorsellum 3.1; lengths mesosoma/gaster 0.9. Wings. CC length/width 22.5; lengths CC/MV 1.1; lengths MV/ST 1.9; lengths MV/ PM 4.8; lengths PM/ST 0.4; submarginal vein with six setae on dorsal surface. Gaster. With lateral tufts of pale and flattened setae on $\mathrm{Gt}_{5-6}$.

\section{Eriastichus ebulis sp. nov.}

http://zoobank.org/44D3C6E8-F596-4AA4-BFD7-DCA4E8D2EC18

Figures 8, 10, 23

Type locality. Costa Rica, San José, San Gerardo de Dota, 9³3'N, 8347'W, 20-21. ii.2013, J.S. Noyes leg.

Type specimen. Holotype male dried and glued to a paper card. Original labels: "COSTA RICA, San José, San Gerardo de Dota, 20-21.ii.2013, J.S. Noyes, NHM (Ent) 2012-91", "HOLOTYPE Eriastichus ebulis Hansson" [red printed label], (NHMUK014431032).

Additional type material. Paratypes $(4 \hat{\jmath} \hat{)}): 2 \hat{\jmath} \hat{\sigma}$ with same label data as holotype (NHMUK014431033, NHMUK014431034); $2 \hat{0}$ đ "COSTA RICA, Cartago, Humo, El Copal, 947'N, 8345'W, 1050-1250 m, 29.ii-6.iii.2008, C. Hansson" (MZLU:7035.2, MZUCR:01693).

Diagnosis (male). Head dark brown, antenna brown; ventral plaque on scape ca. $0.3 \times$ as long as scape (Fig. 23), antenna with dorsobasal setae on F1 $0.5 \times$ as long as F1; gaster with lateral tufts of pale and flattened setae on $\mathrm{Gt}_{5-6}$.

Description (male holotype NHMUK014431032). Length of body $1.5 \mathrm{~mm}$ (paratypes 1.6-2.0 mm). Head dark brown, antenna brown, ventral plaque black. Mesoscutum and mesoscutellum black with metallic tinges, dorsellum and propodeum black. Legs with coxae, femora and $4^{\text {th }}$ tarsomere on all legs dark brown, trochanters, tibiae and tarsomeres 1-4 yellowish brown. Gaster dark brown.

Head. Length/width in frontal view 0.8; width/length in dorsal view 2.0; POL/ OOL 1.5; WM/MS 1.4; MS/HE 0.6; HE/head length in frontal view 0.6; widths head/mesoscutum 1.0. Antenna. Pedicel + flagellum length/mesoscutum width 2.3; pedicel + flagellum length/head width 1.9; lengths scape/ventral plaque 3.0; ventral plaque located in the middle of scape; scape length/width 3.0; lengths scape/head (dorsal view) 0.5; scape length/HE 0.9; length/width F1, F2, F3, F4, clava: 3.2, 3.0, 3.0, 3.0, 7.8; length dorsobasal setae on F1/length F1 0.5. Mesosoma. Length/width 1.8; mesoscutum length/width 0.8 ; mesoscutellum length/width 0.8; widths SMG/SLG 1.6; enclosed space between SMG length/width 2.2; lengths mesoscutum/mesoscutellum 1.6; lengths mesoscutellum/dorsellum 2.7; lengths mesosoma/gaster 0.8. Wings. CC length/width 26.0; lengths CC/MV 1.0; lengths MV/ST 1.9; lengths MV/PM 
7.4; lengths PM/ST 0.3; submarginal vein with seven setae (5-9 setae in paratypes) on dorsal surface. Gaster. With lateral tufts of pale and flattened setae on $\mathrm{Gt}_{5-6}$.

\section{Eriastichus egrestis sp. nov.}

http://zoobank.org/B875A5F8-F524-407E-8D2F-6A874BE758FA

Figure 24

Type locality. Costa Rica, Puntarenas, San Vito, Las Cruces, $8^{\circ} 46^{\prime} \mathrm{N}, 82^{\circ} 57^{\prime} \mathrm{W}$, 1300 m, 15-16.ii.2006, J.S. Noyes leg.

Type specimen. Holotype male dried and glued to a paper card. Original labels: "COSTA RICA, Puntarenas, San Vito, Las Cruces, 846'N, 82 57'W, 1300 m, 15-16. ii.2006, J.S. Noyes", "HOLOTYPE Eriastichus egrestis Hansson" [red printed label], (NHMUK014431035).

Diagnosis (male). Head dark brown, antenna brown; ventral plaque on scape ca. $0.2 \times$ as long as scape (Fig. 24), antenna with dorsobasal setae on F1 $1.3 \times$ as long as F1; gaster with lateral tufts of pale and flattened setae on $\mathrm{Gt}_{6}$.

Description (male holotype NHMUK014431035). Length of body $1.0 \mathrm{~mm}$. Head dark brown, antenna brown, ventral plaque dark brown. Mesoscutum and mesoscutellum dark brown with metallic tinges, dorsellum and propodeum dark brown. Legs with coxae and femora brown, trochanters, tibiae and tarsi yellowish brown. Gaster dark brown.

Head. Length/width in frontal view 0.8 ; width/length in dorsal view 2.5; POL/ OOL 2.4; WM/MS 1.5; MS/HE 0.6; HE/head length in frontal view 0.5; widths head/mesoscutum 1.3. Antenna. Pedicel + flagellum length/mesoscutum width 1.4; pedicel + flagellum length/head width 1.1; lengths scape/ventral plaque 4.5; ventral plaque located below the middle of scape; scape length/width 3.6; lengths scape/ head (dorsal view) 0.5; scape length/HE 0.9; length/width F1, F2, F3, F4, clava: 1.4, 1.6, 1.6, 1.6, 4.3; length dorsobasal setae on F1/length F1 1.3. Mesosoma. Length/ width 1.5; mesoscutum length/width 0.6 ; mesoscutellum length/width 0.6 ; widths SMG/SLG 1.4; enclosed space between SMG length/width 1.5; lengths mesoscutum/ mesoscutellum 1.5; lengths mesoscutellum/dorsellum 2.5; lengths mesosoma/gaster 0.8. Wings. CC length/width 14.5; lengths costal cell/MV 1.0; lengths MV/ST 2.0; lengths MV/PM 3.5; lengths PM/ST 0.6; submarginal vein with five setae on dorsal surface. Gaster. With lateral tufts of pale and flattened setae on $\mathrm{Gt}_{6}$.

\section{Eriastichus eleagnis sp. nov.}

http://zoobank.org/81F12226-5418-40DF-8BD8-D872B5B814B4

Figures 1, 27

Type locality. Costa Rica, Puntarenas, Estación Biológica Monteverde, $10^{\circ} 20^{\prime} \mathrm{N}$, 84²9'W, 1540 m, 18-25.ii.2004, C. Hansson leg. 
Type specimen. Holotype male dried and glued to a paper card. Original labels: "COSTA RICA, Puntarenas, E.B. Monteverde, $10^{\circ} 20^{\prime} \mathrm{N}, 84^{\circ} 49^{\prime} \mathrm{W}, 1540$ m, 18-25. ii.2004, C. Hansson", "HOLOTYPE Eriastichus eleagnis Hansson" [red printed label], (MZLU:7036.1).

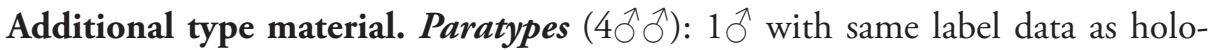
type (MZLU:7036.2); 10 from same locality as holotype but collected 26.ii.2007 (NHMUK014431036); 10 "COSTA RICA, Puntarenas, San Vito, E.B. Las Alturas, $1500 \mathrm{~m}, 8^{\circ} 57^{\prime} \mathrm{N}, 82^{\circ} 50^{\prime} \mathrm{W}, 17-18 . i i .2012$, J.S. Noyes, NHM (Ent) 2012-91" (NHMUK014431037); 10 “COSTA RICA, San José, San Gerardo de Dota, 20-21. ii.2013, J.S. Noyes, NHM (Ent) 2012-91” (NHMUK014431038).

Diagnosis (male). Head dark brown, scape yellowish brown, pedicel and flagellum brown; ventral plaque on scape ca. $0.4 \times$ as long as scape (Fig. 27), antenna with dorsobasal setae on F1 $1.5 \times$ as long as F1; gaster with lateral tufts of pale and flattened setae on $\mathrm{Gt}_{6}$.

Description (male holotype MZLU:7036.1). Length of body $1.4 \mathrm{~mm}$ (paratypes 1.3-1.5 mm). Head dark brown, scape yellowish brown, pedicel and flagellum brown, ventral plaque dark brown. Mesoscutum, mesoscutellum and propodeum dark brown, dorsellum pale brown. Legs with coxae and femora brown, trochanters, tibiae and tarsi yellowish brown. Gaster dark brown.

Head. Length/width in frontal view 0.8; width/length in dorsal view 2.4; POL/ OOL 2.4; WM/MS 1.5; MS/HE 0.5; HE/head length in frontal view 0.6; widths head/mesoscutum 1.1. Antenna. Pedicel + flagellum length/mesoscutum width 1.8; pedicel + flagellum length/head width 1.7 ; lengths scape/ventral plaque 2.8 ; ventral plaque located below the middle of scape; scape length/width 3.1; lengths scape/head (dorsal view) 0.6; scape length/HE 1.0; length/width F1, F2, F3, F4, clava: 2.6, 3.0, 3.0, 2.8, 7.6; length dorsobasal setae on F1/length F1 1.5. Mesosoma. Length/width 1.4; mesoscutum length/width 0.6; mesoscutellum length/width 0.8; widths SMG/ SLG 1.1; enclosed space between SMG length/width 2.1; lengths mesoscutum/mesoscutellum 1.6; lengths mesoscutellum/dorsellum 2.9; lengths mesosoma/gaster 0.8. Wings. CC length/width 24.0; lengths CC/MV 1.1; lengths MV/ST 1.9; lengths MV/ PM 3.8; lengths PM/ST 0.5; submarginal vein with seven setae (5-7 setae in paratypes) on dorsal surface. Gaster. With lateral tufts of pale and flattened setae on $\mathrm{Gt}_{6}$.

\section{Eriastichus ellipsis sp. nov.}

http://zoobank.org/B8933986-9084-433F-B5BC-3BB531D04D8E

Figure 25

Type locality. Costa Rica, Heredia, Estación Biológica La Selva, 75 m, 10 $26^{\prime} \mathrm{N}$, $84^{\circ} 01^{\prime} \mathrm{W}, 24-25.1 i .2011$, J.S. Noyes leg.

Type specimen. Holotype male dried and glued to a paper card. Original labels: "COSTA RICA, Heredia, E.B. La Selva, 75 m, $10^{\circ} 26^{\prime} \mathrm{N}, 84^{\circ} 01^{\prime} \mathrm{W}, 24-25 . i i .2011$, 
J.S. Noyes, NHM (Ent) 2011-93”, “HOLOTYPE Eriastichus ellipsis Hansson” [red printed label], (NHMUK014431039).

Additional type material. Paratype $1 \hat{\sigma}$ with same label data as holotype (NHMUK014431040).

Diagnosis (male). Head dark brown with part below antennal toruli yellowish brown, scape yellowish brown, pedicel and flagellum brown; ventral plaque on scape ca. $0.2 \times$ as long as scape (Fig. 25), antenna with dorsobasal setae on F1 $0.6 \times$ as long as F1; gaster with lateral tufts of pale and flattened setae on $\mathrm{Gt}_{6}$.

Description (male holotype NHMUK014431039). Length of body $1.1 \mathrm{~mm}$ (paratype $1.1 \mathrm{~mm}$ ). Head dark brown with part below antennal toruli yellowish brown, scape yellowish brown, pedicel and flagellum brown, ventral plaque dark brown. Mesoscutum and mesoscutellum black with metallic tinges, dorsellum pale brown, propodeum dark brown. Legs yellowish brown. Gaster dark brown.

Head. Length/width in frontal view 0.7; width/length in dorsal view 2.3; POL/ OOL 3.5; WM/MS 1.3; MS/HE 0.5; HE/head length in frontal view 0.6; widths head/mesoscutum 1.2. Antenna. Pedicel + flagellum length/mesoscutum width 1.8; pedicel + flagellum length/head width 1.5 ; lengths scape/ventral plaque 4.8 ; ventral plaque located in the middle of scape; scape length/width 3.2; lengths scape/head (dorsal view) 0.5; scape length/HE 0.8; length/width F1, F2, F3, F4, clava: 1.5, 2.0, 2.2, 2.2, 6.0; length dorsobasal setae on F1/length F1 0.6. Mesosoma. Length/width 1.3; mesoscutum length/width 0.6; mesoscutellum length/width 0.7 ; widths SMG/SLG 1.4; enclosed space between SMG length/width 1.7; lengths mesoscutum/mesoscutellum 1.5; lengths mesoscutellum/dorsellum 2.8; lengths mesosoma/gaster 0.8. Wings. CC length/width 21.3; lengths CC/MV 1.1; lengths MV/ST 2.0; lengths MV/PM 5.0; lengths PM/ST 0.4; submarginal vein with six setae (five setae in paratype) on dorsal surface. Gaster. With lateral tufts of pale and flattened setae on $\mathrm{Gt}_{6}$.

\section{Eriastichus eminis sp. nov.}

http://zoobank.org/91B5BBE0-7453-4F7D-BB97-FD1DFC02224F

Figure 31

Type locality. Costa Rica, Guanacaste, Estación Pitilla, Area Conservación de Guanacaste, $10^{\circ} 59^{\prime} \mathrm{N}, 85^{\circ} 26^{\prime} \mathrm{W}, 21 . i 1.2011$, J.S. Noyes leg.

Type specimen. Holotype male dried and glued to a paper card. Original labels: "COSTA RICA, Guanacaste, Est. Pitilla, ACG, 21.ii.2011, J.S. Noyes, NHM (Ent) 2011-93", “HOLOTYPE Eriastichus eminis Hansson" [red printed label], (NHMUK014431041).

Diagnosis (male). Head dark brown with part below antennal toruli dark yellowish brown, scape yellowish brown, pedicel and flagellum brown; ventral plaque on scape ca. $0.2 \times$ as long as scape (Fig. 31), antenna with dorsobasal setae on F1 $1.1 \times$ as long as F1; gaster with lateral tufts of pale and flattened setae on $\mathrm{Gt}_{6}$. 
Description (male holotype NHMUK014431041). Length of body $1.2 \mathrm{~mm}$. Head dark brown with part below antennal toruli dark yellowish brown, scape yellowish brown, pedicel and flagellum brown, ventral plaque dark brown. Mesoscutum, mesoscutellum and propodeum dark brown, dorsellum pale brown. Legs yellowish brown. Gaster dark brown.

Head. Length/width in frontal view 0.8 ; width/length in dorsal view 2.2; POL/ OOL 2.2; WM/MS 1.5; MS/HE 0.5; HE/head length in frontal view 0.6; widths head/mesoscutum 1.2. Antenna. Pedicel + flagellum length/mesoscutum width 2.4; pedicel + flagellum length/head width 1.9; lengths scape/ventral plaque 4.5; ventral plaque located below the middle of scape; scape length/width 2.9; lengths scape/head (dorsal view) 0.5; scape length/HE 0.8; length/width F1, F2, F3, F4, clava: 3.5, 3.5, 3.3, 3.5, 8.0; length dorsobasal setae on F1/length F1 1.1. Mesosoma. Length/width 1.7; mesoscutum length/width 0.7; mesoscutellum length/width 0.9; widths SMG/ SLG 1.3; enclosed space between SMG length/width 2.2; lengths mesoscutum/mesoscutellum 1.5; lengths mesoscutellum/dorsellum 2.9; lengths mesosoma/gaster 0.8. Wings. CC length/width 26.3; lengths CC/MV 1.1; lengths MV/ST 2.1; lengths MV/ PM 5.7; lengths PM/ST 0.4; submarginal vein with six setae on dorsal surface. Gaster. With lateral tufts of pale and flattened setae on $\mathrm{Gt}_{6}$.

\section{Eriastichus facilis sp. nov.}

http://zoobank.org/1759534A-5BB1-4670-8391-9AD918D65A24

Figure 28

Type locality. Costa Rica, Puntarenas, Estación Biológica Monteverde, $10^{\circ} 20^{\prime} \mathrm{N}$, $84^{\circ} 49^{\prime} \mathrm{W}, 1540 \mathrm{~m}, 26 . i \mathrm{i} .2007$, J.S. Noyes leg.

Type specimen. Holotype male dried and glued to a paper card. Original labels: "COSTA RICA, Puntarenas, E.B. Monteverde, $10^{\circ} 20^{\prime} \mathrm{N}, 84^{\circ} 49^{\prime} \mathrm{W}, 1540 \mathrm{~m}$, 26.ii.2007, J.S. Noyes", "HOLOTYPE Eriastichus facilis Hansson" [red printed label], (NHMUK014431042).

Additional type material. Paratypes $(2 \widehat{\jmath}): 1 \overbrace{}^{\Uparrow}$ "COSTA RICA, Cartago, Humo, El Copal, 947'N, 8345'W, 1050-1250 m, 29.ii-6.iii.2008, C. Hansson” (MZLU:7037.2); $10^{\lambda}$ “COSTA RICA, Alajuela, Est. Pilón, $10^{\circ} 43^{\prime} \mathrm{N}, 85^{\circ} 59^{\prime} \mathrm{W}, 700 \mathrm{~m}$, 12-18.ii.2003, C. Hansson \& J.A. Azofeifa" (MZLU:7037.3).

Diagnosis (male). Head dark brown, scape yellowish brown, pedicel and flagellum brown; ventral plaque on scape ca. $0.2 \times$ as long as scape (Fig. 28), antenna with dorsobasal setae on F1 $0.9 \times$ as long as F1; gaster with lateral tufts of pale and flattened setae on Gt ${ }_{6}$.

Description (male holotype NHMUK014431042). Length of body $1.5 \mathrm{~mm}$ (paratypes 1.0-1.1 mm). Head dark brown, scape yellowish brown, pedicel and flagellum brown, ventral plaque dark brown. Mesoscutum, mesoscutellum and propodeum dark brown, dorsellum pale brown. Legs with hind coxa and hind femur dark brown, remaining parts of legs yellowish brown. Gaster dark brown.

Head. Length/width in frontal view 0.8; width/length in dorsal view 2.4; POL/ OOL 2.6; WM/MS 1.4; MS/HE 0.5; HE/head length in frontal view 0.6; widths 


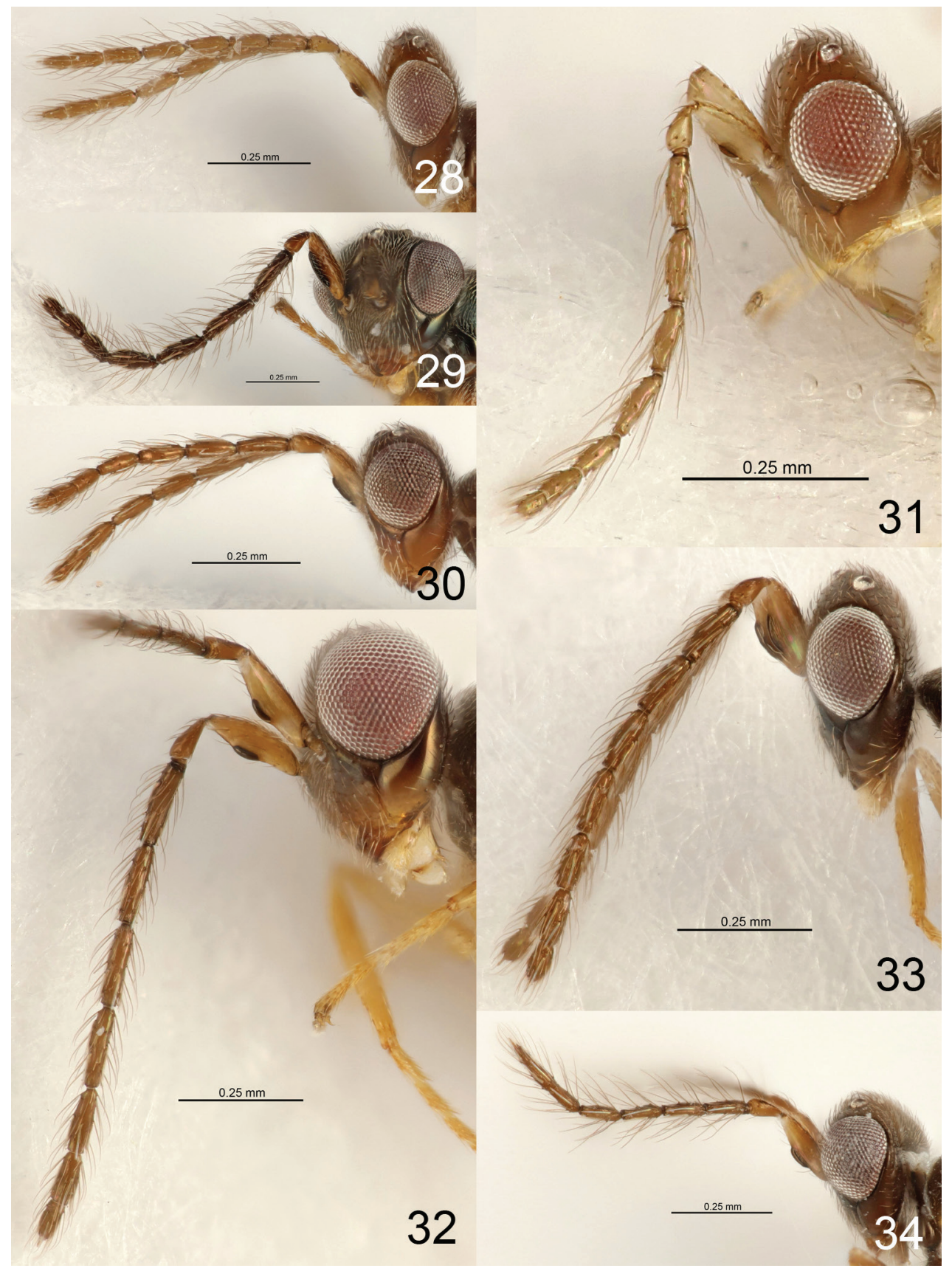

Figures 28-34. Eriastichus spp. head and antenna in lateral view, male holotypes 28 E. facilis 29 E. fenestris $\mathbf{3 0}$ E. follis $\mathbf{3} 1$ E. eminis $\mathbf{3 2}$ E. glanis $\mathbf{3 3}$ E. geratis $\mathbf{3 4}$ E. galeatis.

head/mesoscutum 1.0. Antenna. Pedicel + flagellum length/mesoscutum width 1.7; pedicel + flagellum length/head width 1.6; lengths scape/ventral plaque 4.7; ventral plaque located below the middle of scape; scape length/width 3.5; lengths scape/head 
(dorsal view) 0.5; scape length/HE 0.9; length/width F1, F2, F3, F4, clava: 2.2, 2.2, 2.5, 2.5, 6.3; length dorsobasal setae on F1/length F1 0.9. Mesosoma. Length/width 1.4; mesoscutum length/width 0.6; mesoscutellum length/width 0.7; widths SMG/ SLG 1.2; enclosed space between SMG length/width 2.1; lengths mesoscutum/mesoscutellum 1.4; lengths mesoscutellum/dorsellum 2.7; lengths mesosoma/gaster 0.9. Wings. CC length/width 18.0; lengths CC/MV 1.2; lengths MV/ST 2.0; lengths MV/ PM 3.8; lengths PM/ST 0.5; submarginal vein with five setae on dorsal surface, in all types. Gaster. With lateral tufts of pale and flattened setae on $\mathrm{Gt}_{6}$.

\section{Eriastichus fenestris sp. nov.}

http://zoobank.org/68B54220-6BD6-40CE-9CC0-CEEE6962E631

Figures 5, 29

Type locality. Costa Rica, San José, San Gerardo de Dota, 9³3'N, 834ㄱ'W, 20-21. ii.2013, J.S. Noyes leg.

Type specimen. Holotype male dried and glued to a paper card. Original labels: "COSTA RICA, San José, San Gerardo de Dota, 20-21.ii.2013, J.S. Noyes, NHM (Ent) 2012-91", "HOLOTYPE Eriastichus fenestris Hansson" [red printed label], (NHMUK014431043).

Diagnosis (male). Head black, scape brown, pedicel and flagellum dark brown; ventral plaque on scape ca. $0.6 \times$ as long as scape (Fig. 29), antenna with dorsobasal setae on F1 $0.7 \times$ as long as F1; gaster with lateral tufts of pale and flattened setae on $\mathrm{Gt}_{4-6}$.

Description (male holotype NHMUK014431043). Length of body $2.1 \mathrm{~mm}$. Head black, scape brown, pedicel, flagellum and ventral plaque dark brown. Mesoscutum, mesoscutellum and dorsellum black with metallic tinges, propodeum black. Legs with fore and mid coxae yellowish brown with base dark brown, hind coxa black, remaining parts of legs yellowish brown. Gaster dark brown.

Head. Length/width in frontal view 0.7 ; width/length in dorsal view 2.2; POL/ OOL 1.8; WM/MS 1.6; MS/HE 0.6; HE/head length in frontal view 0.6; widths head/mesoscutum 1.0. Antenna. Pedicel + flagellum length/mesoscutum width 2.1; pedicel + flagellum length/head width 2.1; lengths scape/ventral plaque 1.7; ventral plaque located in the middle of scape; scape length/width 2.6; lengths scape/head (dorsal view) 0.5; scape length/HE 0.9; length/width F1, F2, F3, F4, clava: 3.2, 3.0, 2.9, 3.0, 5.8; length dorsobasal setae on F1/length F1 0.7. Mesosoma. Length/width 1.6; mesoscutum length/width 0.6; mesoscutellum length/width 0.8; widths SMG/SLG 1.2; enclosed space between SMG length/width 2.2; lengths mesoscutum/mesoscutellum 1.5; lengths mesoscutellum/dorsellum 2.3; lengths mesosoma/gaster 1.0. Wings. CC length/width 19.0; lengths CC/MV 0.9; lengths MV/ST 2.1; lengths MV/PM 5.5; lengths PM/ST 0.4; submarginal vein with ten setae on dorsal surface. Gaster. With lateral tufts of pale and flattened setae on $\mathrm{Gt}_{4-6}$. 


\section{Eriastichus follis sp. nov.}

http://zoobank.org/F9EFCF3E-87E4-4157-9AE2-ADDA7AF0E7D3

Figure 30

Type locality. Costa Rica, Puntarenas, Estación Biológica Monteverde, $10^{\circ} 20^{\prime} \mathrm{N}$, $84^{\circ} 49^{\prime} \mathrm{W}, 1540 \mathrm{~m}, 26 . \mathrm{ii} .2007$, J.S. Noyes leg.

Type specimen. Holotype male dried and glued to a paper card. Original labels: "COSTA RICA, Puntarenas, E.B. Monteverde, $10^{\circ} 20^{\prime} \mathrm{N}, 84^{\circ} 49^{\prime} \mathrm{W}, 1540 \mathrm{~m}$, 26.ii.2007, J.S. Noyes", "HOLOTYPE Eriastichus follis Hansson" [red printed label], (NHMUK014431044).

Diagnosis (male). Head dark brown with part below antennal toruli pale brown, scape and pedicel pale brown, flagellum brown; ventral plaque on scape ca. $0.6 \times$ as long as scape (Fig. 30), antenna with dorsobasal setae on F1 $0.6 \times$ as long as F1; gaster with lateral tufts of pale and flattened setae on $\mathrm{Gt}_{6}$.

Description (male holotype NHMUK014431044). Length of body $1.5 \mathrm{~mm}$. Head dark brown with part below antennal toruli pale brown, scape and pedicel pale brown, flagellum brown, ventral plaque dark brown. Mesoscutum and mesoscutellum dark brown with metallic tinges, dorsellum and propodeum dark brown. Legs with hind coxa pale brown, remaining parts of legs yellowish brown. Gaster dark brown.

Head. Length/width in frontal view 0.8 ; width/length in dorsal view 2.1; POL/ OOL 1.9; WM/MS 1.2; MS/HE 0.5; HE/head length in frontal view 0.5; widths head/mesoscutum 1.2. Antenna. Pedicel + flagellum length/mesoscutum width 2.1; pedicel + flagellum length/head width 1.8 ; lengths scape/ventral plaque 1.7; ventral plaque located below the middle of scape; scape length/width 2.9; lengths scape/head (dorsal view) 0.5; scape length/HE 0.8; length/width F1, F2, F3, F4, clava: 2.8, 2.7, 2.7, 2.7, 6.3; length dorsobasal setae on F1/length F1 0.6. Mesosoma. Length/width 1.7; mesoscutum length/width 0.7; mesoscutellum length/width 0.8; widths SMG/ SLG 1.2; enclosed space between SMG length/width 2.3; lengths mesoscutum/mesoscutellum 1.5; lengths mesoscutellum/dorsellum 2.8; lengths mesosoma/gaster 0.8. Wings. CC length/width 16.7; lengths CC/MV 0.9; lengths MV/ST 2.3; lengths MV/PM 4.9; lengths PM/ST 0.5; submarginal vein with nine setae on dorsal surface. Gaster. With lateral tufts of pale and flattened setae on $\mathrm{Gt}_{6}$.

\section{Eriastichus galeatis sp. nov.}

http://zoobank.org/04AE8D37-56D4-411C-88A0-04FDC46979A3

Figure 34

Type locality. Costa Rica, Puntarenas, Estación Biológica Monteverde, $10^{\circ} 20^{\prime} \mathrm{N}$, $84^{\circ} 49^{\prime} \mathrm{W}, 1540 \mathrm{~m}, 26 . i i .2007$, J.S. Noyes leg.

Type specimen. Holotype male dried and glued to a paper card. Original labels: "COSTA RICA, Puntarenas, E.B. Monteverde, $10^{\circ} 20^{\prime} \mathrm{N}, 84^{\circ} 49^{\prime} \mathrm{W}, 1540 \mathrm{~m}$, 
26.ii.2007, J.S. Noyes", "HOLOTYPE Eriastichus galeatis Hansson” [red printed label], (NHMUK014431045).

Diagnosis (male). Head dark brown, scape and pedicel pale brown, flagellum brown; ventral plaque on scape ca. $0.3 \times$ as long as scape (Fig. 34), antenna with dorsobasal setae on F1 $1.0 \times$ as long as F1; gaster with lateral tufts of pale and flattened setae on $\mathrm{Gt}_{6}$.

Description (male holotype NHMUK014431045). Length of body $1.5 \mathrm{~mm}$. Head dark brown, scape and pedicel pale brown, flagellum brown, ventral plaque dark brown. Mesoscutum, mesoscutellum, dorsellum and propodeum black with metallic tinges. Legs with coxae and femora dark brown, hind tibia pale brown, remaining parts of legs yellowish brown. Gaster dark brown.

Head. Length/width in frontal view 0.7 ; width/length in dorsal view 2.3; POL/ OOL 2.1; WM/MS 1.6; MS/HE 0.5; HE/head length in frontal view 0.6; widths head/mesoscutum 1.1. Antenna. Pedicel + flagellum length/mesoscutum width 1.8; pedicel + flagellum length/head width 1.7; lengths scape/ventral plaque 3.1; ventral plaque located below the middle of scape; scape length/width 3.3; lengths scape/head (dorsal view) 0.5; scape length/HE 0.9; length/width F1, F2, F3, F4, clava: 3.0, 2.5, 2.5, 2.5, 6.3; length dorsobasal setae on F1/length F1 1.0. Mesosoma. Length/width 1.5; mesoscutum length/width 0.6; mesoscutellum length/width 0.7; widths SMG/ SLG 1.3; enclosed space between SMG length/width 1.9; lengths mesoscutum/mesoscutellum 1.5; lengths mesoscutellum/dorsellum 2.7; lengths mesosoma/gaster 0.9. Wings. CC length/width 19.2; lengths CC/MV 1.0; lengths MV/ST 2.2; lengths MV/ PM 3.8; lengths PM/ST 0.6; submarginal vein with eight setae on dorsal surface. Gaster. With lateral tufts of pale and flattened setae on $\mathrm{Gt}_{6}$.

\section{Eriastichus geratis sp. nov.}

http://zoobank.org/9BF19DD1-E099-457E-AFA4-0D5D82E0BA08

Figure 33

Type locality. Costa Rica, Puntarenas, San Vito, Estación Biológica Las Alturas, 1500 $\mathrm{m}, 8^{\circ} 57^{\prime} \mathrm{N}, 82^{\circ} 50^{\prime} \mathrm{W}, 17-18 . i i .2012$, J.S. Noyes leg.

Type specimen. Holotype male dried and glued to a paper card. Original labels: "COSTA RICA, Puntarenas, San Vito, E.B. Las Alturas, 1500 m, 8 $57^{\prime} \mathrm{N}, 82^{\circ} 50^{\prime} \mathrm{W}$, 17-18.ii.2012, J.S. Noyes, NHM (Ent) 2012-91", "HOLOTYPE Eriastichus geratis Hansson" [red printed label], (NHMUK014431046).

Additional type material. Paratype $1 \widehat{\partial}^{\lambda}$ "COSTA RICA, San José, San Gerardo de Dota, 20-21.ii.2013, J.S. Noyes, NHM (Ent) 2012-91” (NHMUK014431047).

Diagnosis (male). Head dark brown, scape and pedicel pale brown, flagellum brown; ventral plaque on scape ca. $0.4 \times$ as long as scape (Fig. 33), antenna with dorsobasal setae on F1 $0.6 \times$ as long as F1; gaster with lateral tufts of pale and flattened setae on $\mathrm{Gt}_{6}$. 
Description (male holotype NHMUK014431046). Length of body $1.8 \mathrm{~mm}$ (paratype $1.7 \mathrm{~mm}$ ). Head dark brown, scape and pedicel pale brown, flagellum brown, ventral plaque dark brown. Mesoscutum and mesoscutellum black with metallic tinges, dorsellum dark brown, propodeum black. Legs with coxae, mid and hind femora dark brown, fore femur pale brown, remaining parts of legs yellowish brown. Gaster dark brown.

Head. Length/width in frontal view 0.8; width/length in dorsal view 2.4; POL/ OOL 1.9; WM/MS 1.6; MS/HE 0.6; HE/head length in frontal view 0.5; widths head/mesoscutum 1.0. Antenna. Pedicel + flagellum length/mesoscutum width 1.8; pedicel + flagellum length/head width 1.8; lengths scape/ventral plaque 2.5; ventral plaque located in the middle of scape; scape length/width 2.8; lengths scape/head (dorsal view) 0.5; scape length/HE 0.9; length/width F1, F2, F3, F4, clava: 3.2, 2.8, 3.2, 3.0, 7.7; length dorsobasal setae on F1/length F1 0.6. Mesosoma. Length/width 1.5; mesoscutum length/width 0.6; mesoscutellum length/width 0.7; widths SMG/SLG 1.2; enclosed space between SMG length/width 1.9; lengths mesoscutum/mesoscutellum 1.5; lengths mesoscutellum/dorsellum 2.5; lengths mesosoma/gaster 0.8. Wings. CC length/width 19.3; lengths CC/MV 1.1; lengths MV/ST 2.2; lengths MV/PM 4.3; lengths PM/ST 0.5; submarginal vein with six setae on dorsal surface, in both types. Gaster. With lateral tufts of pale and flattened setae on $\mathrm{Gt}_{6}$.

\section{Eriastichus glanis sp. nov.}

http://zoobank.org/CC8A526B-4C9D-4B48-8503-9CE11D084C54

Figure 32

Type locality. Costa Rica, Limón, Reserva Biológica Hitoy-Cerere, Headquarters, $100 \mathrm{~m}, 9^{\circ} 40^{\prime} \mathrm{N}, 83^{\circ} 02^{\prime} \mathrm{W}, 24-26 . i i .2008$, J.S. Noyes leg.

Type specimen. Holotype male dried and glued to a paper card. Original labels: "COSTA RICA, Limón, R.B. Hitoy-Cerere, HQ, 100 m, 940'N, 8302'W, 24-26. ii.2008, J.S. Noyes, BMNH(Ent) 2010-21", "HOLOTYPE Eriastichus glanis Hansson" [red printed label], (NHMUK014431048).

Diagnosis (male). Head dark brown, scape yellowish brown, pedicel pale brown, flagellum brown; ventral plaque on scape ca. $0.3 \times$ as long as scape (Fig. 32), antenna with dorsobasal setae on F1 $0.5 \times$ as long as F1; gaster with lateral tufts of pale and flattened setae on $\mathrm{Gt}_{6}$.

Description (male holotype NHMUK014431048). Length of body $2.0 \mathrm{~mm}$. Head dark brown, scape yellowish brown, pedicel pale brown, flagellum brown, ventral plaque dark brown. Mesoscutum and mesoscutellum black with metallic tinges, dorsellum and propodeum dark brown. Legs yellowish brown, hind coxa with base dark brown. Gaster dark brown.

Head. Length/width in frontal view 0.7; width/length in dorsal view 2.1; POL/ OOL 1.3; WM/MS 1.8; MS/HE 0.4; HE/head length in frontal view 0.6; widths 
head/mesoscutum 1.0. Antenna. Pedicel + flagellum length/mesoscutum width 2.4; pedicel + flagellum length/head width 2.0; lengths scape/ventral plaque 4.0; ventral plaque located in the middle of scape; scape length/width 3.1; lengths scape/head (dorsal view) 0.5 ; scape length/HE 0.8; length/width F1, F2, F3, F4, clava: 4.0, 4.0, 4.2, 3.8, 8.2; length dorsobasal setae on F1/length F1 0.5. Mesosoma. Length/width 1.6; mesoscutum length/width 0.7; mesoscutellum length/width 0.9; widths SMG/SLG 1.7; enclosed space between SMG length/width 2.2; lengths mesoscutum/mesoscutellum 1.4; lengths mesoscutellum/dorsellum 3.3; lengths mesosoma/gaster 0.7. Wings. CC length/width 18.7; lengths CC/MV 0.8; lengths MV/ST 2.9; lengths MV/PM 4.7; lengths PM/ST 0.6; submarginal vein with nine setae on dorsal surface. Gaster. With lateral tufts of pale and flattened setae on $\mathrm{Gt}_{6}$.

\section{Eriastichus hilaris sp. nov.}

http://zoobank.org/9FFEC823-0436-45EE-BA8F-54FD1FB5E740

Figure 35

Type locality. Costa Rica, San José, San Gerardo de Dota, 9³3'N, 8347'W, 20-21. ii.2013, J.S. Noyes leg.

Type specimen. Holotype male dried and glued to a paper card. Original labels: "COSTA RICA, San José, San Gerardo de Dota, 20-21.ii.2013, J.S. Noyes, NHM (Ent) 2012-91", "HOLOTYPE Eriastichus hilaris Hansson" [red printed label], (NHMUK014431049).

Diagnosis (male). Head black with part below antennal toruli pale brown, scape yellowish brown, pedicel pale brown, flagellum dark brown; ventral plaque on scape ca. $0.5 \times$ as long as scape (Fig. 35), antenna with dorsobasal setae on F1 $0.4 \times$ as long as F1; gaster with lateral tufts of pale and flattened setae on $\mathrm{Gt}_{5-6}$.

Description (male holotype NHMUK014431049). Length of body $1.7 \mathrm{~mm}$. Head black with part below antennal toruli pale brown, scape yellowish brown, pedicel pale brown, flagellum dark brown, ventral plaque dark brown. Mesoscutum and mesoscutellum black with metallic tinges, dorsellum dark brown, propodeum black. Legs with hind coxa with basal $1 / 2$ dark brown and apical $1 / 2$ yellowish brown, remaining parts of legs yellowish brown. Gaster dark brown.

Head. Length/width in frontal view 0.8; width/length in dorsal view 2.0; POL/ OOL 2.0; WM/MS 1.4; MS/HE 0.6; HE/head length in frontal view 0.5; widths head/mesoscutum 1.2. Antenna. Pedicel + flagellum length/mesoscutum width 1.9; pedicel + flagellum length/head width 1.7; lengths scape/ventral plaque 2.2; ventral plaque located above the middle of scape; scape length/width 2.9; lengths scape/head (dorsal view) 0.5; scape length/HE 0.9; length/width F1, F2, F3, F4, clava: 2.3, 2.4, 2.1, 2.2, 4.9; length dorsobasal setae on F1/length F1 0.4. Mesosoma. Length/width 1.6; mesoscutum length/width 0.5; mesoscutellum length/width 0.8 ; widths SMG/ SLG 1.0; enclosed space between SMG length/width 2.4; lengths mesoscutum/mesoscutellum 1.2; lengths mesoscutellum/dorsellum 2.9; lengths mesosoma/gaster 0.8. Wings. CC length/width 17.3; lengths CC/MV 1.0; lengths MV/ST 2.2; lengths 
MV/PM 4.5; lengths PM/ST 0.5; submarginal vein with nine setae on dorsal surface. Gaster. With lateral tufts of pale and flattened setae on $\mathrm{Gt}_{5-6^{\circ}}$.

\section{Eriastichus johnlasallei sp. nov.}

http://zoobank.org/2A25525C-B5A5-471F-9697-A6936C8FB55E

Figure 37

Type locality. Costa Rica, Cartago, Humo, El Copal, 947'N, 8345'W, 1050 1250 m, 29.ii-6.iii.2008, C. Hansson leg.

Type specimen. Holotype male dried and glued to a paper card. Original labels: "COSTA RICA, Cartago, Humo, El Copal, 947'N, 8345'W, 1050-1250 m, 29.ii6.iii.2008, C. Hansson", "HOLOTYPE Eriastichus johnlasallei Hansson” [red printed label], (MZLU:7038.1).

Diagnosis (male). Head and antenna dark brown; ventral plaque on scape ca. 0.2 $\times$ as long as scape (Fig. 37), antenna with dorsobasal setae on F1 $0.8 \times$ as long as F1; gaster with lateral tufts of pale and flattened setae on $\mathrm{Gt}_{6}$.

Description (male holotype MZLU:7038.1). Length of body $1.5 \mathrm{~mm}$. Head and antenna dark brown, ventral plaque black. Mesoscutum, mesoscutellum and propodeum dark brown; dorsellum pale brown. Legs yellowish brown, coxae and hind femur brown. Gaster dark brown.

Head. Length/width in frontal view 0.7; width/length in dorsal view 2.3; POL/ OOL 2.0; WM/MS 1.7; MS/HE 0.5; HE/head length in frontal view 0.6; widths head/mesoscutum 1.2. Antenna. Pedicel + flagellum length/mesoscutum width 2.0; pedicel + flagellum length/head width 1.7; lengths scape/ventral plaque 4.7; ventral plaque located in the middle of scape; scape length/width 3.5; lengths scape/head (dorsal view) 0.6; scape length/HE 0.9; length/width F1, F2, F3, F4, clava: 2.5, 2.7, 2.8, 2.8, 6.2; length dorsobasal setae on F1/length F1 0.8. Mesosoma. Length/width 1.5; mesoscutum length/width 0.5 ; mesoscutellum length/width 0.8 ; widths SMG/SLG 1.6; enclosed space between SMG length/width 1.9; lengths mesoscutum/mesoscutellum 1.2; lengths mesoscutellum/dorsellum 3.6; lengths mesosoma/gaster 0.9. Wings. CC length/width 24.0; lengths CC/MV 0.9; lengths MV/ST 2.2; lengths MV/PM 4.7; lengths PM/ST 0.5; submarginal vein with seven setae on dorsal surface. Gaster. With lateral tufts of pale and flattened setae on $\mathrm{Gt}_{6}$.

Etymology. Named after John La Salle, fellow eulophid taxonomist who described Eriastichus.

\section{Eriastichus jobnnoyesi sp. nov.}

http://zoobank.org/1F78E014-A16C-4D6F-8CFB-FC8EEF163665

Figure 40

Type locality. Costa Rica, Heredia, Estación Biológica La Selva, 75 m, 10²6'N, $84^{\circ} 01^{\prime} \mathrm{W}, 24-25 . \mathrm{ii} .2011$, J.S. Noyes leg. 


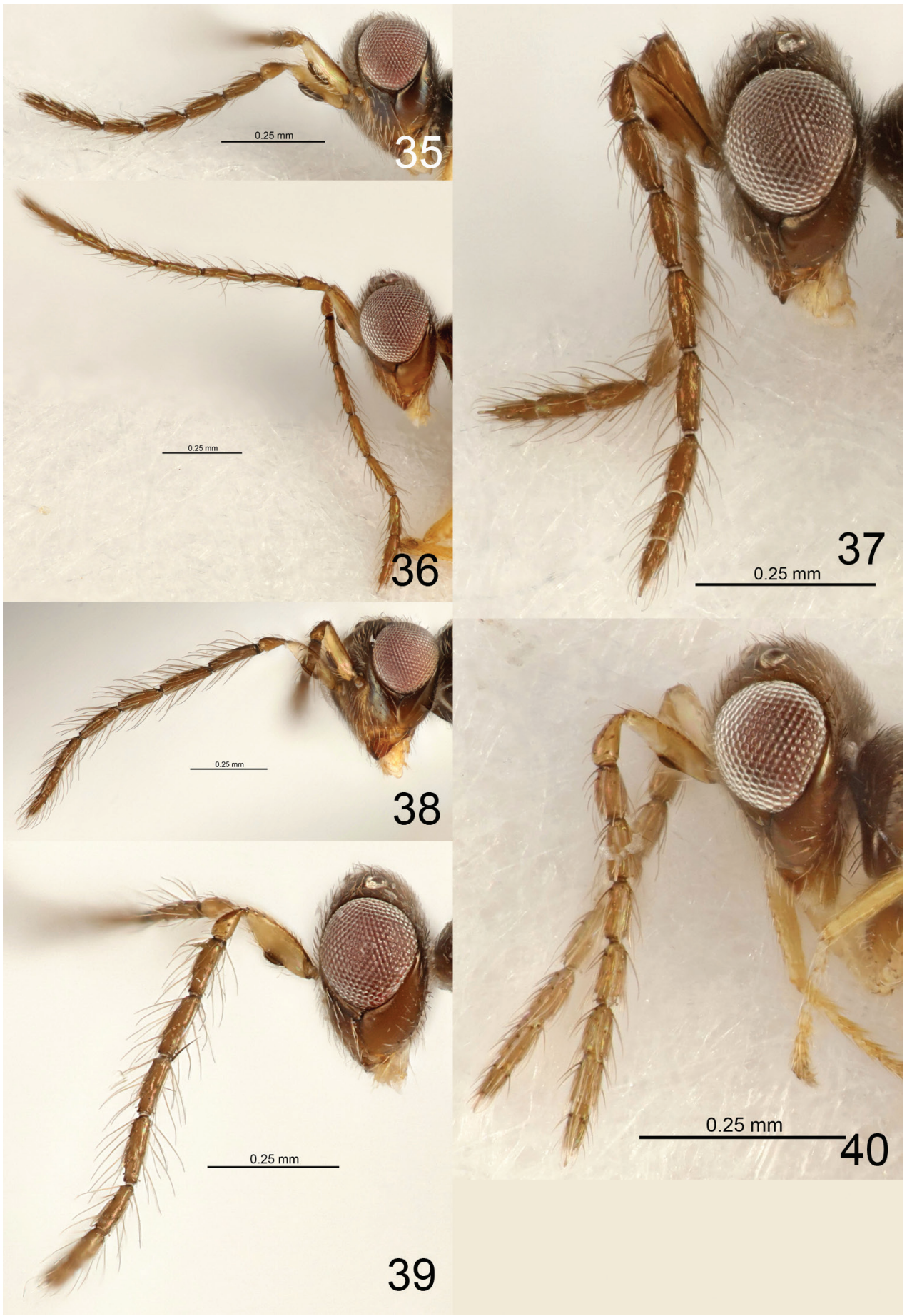

Figures 35-40. Eriastichus spp. head and antenna in lateral view, male holotypes 35 E. hilaris 36 E. maniatis $\mathbf{3 7}$ E. johnlasallei $\mathbf{3 8}$ E. nebulis $\mathbf{3 9}$ E. neonis $\mathbf{4 0}$ E. johnnoyesi. 
Type specimen. Holotype male dried and glued to a paper card. Original labels:

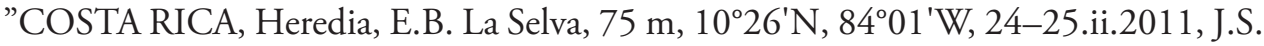
Noyes, NHM (Ent) 2011-93", "HOLOTYPE Eriastichus johnnoyesi Hansson" [red printed label], (NHMUK014431050.

Additional type material. Paratypes $2 \hat{\jmath} \hat{0}$ from same locality as holo-

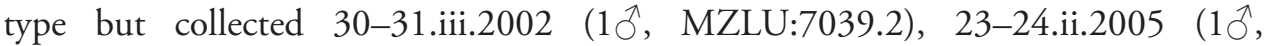
NHMUK014431051).

Diagnosis (male). Head dark brown, scape yellowish brown, pedicel and flagellum brown; ventral plaque on scape ca. $0.2 \times$ as long as scape (Fig. 40), antenna with dorsobasal setae on F1 $0.6 \times$ as long as F1; gaster with lateral tufts of pale and flattened setae on $\mathrm{Gt}_{6}$.

Description (male holotype NHMUK014431050). Length of body $1.1 \mathrm{~mm}$ (paratypes $1.0 \mathrm{~mm}$ ). Head dark brown, scape yellowish brown, pedicel and flagellum brown, ventral plaque dark brown. Mesoscutum and mesoscutellum black, dorsellum and propodeum dark brown. Legs with coxae and hind femur dark brown, remaining parts of legs yellowish brown. Gaster dark brown.

Head. Length/width in frontal view 0.7 ; width/length in dorsal view 2.2; POL/ OOL 3.0; WM/MS 1.3; MS/HE 0.5; HE/head length in frontal view 0.6; widths head/mesoscutum 1.2. Antenna. Pedicel + flagellum length/mesoscutum width 1.8; pedicel + flagellum length/head width 1.6; lengths scape/ventral plaque 5.0; ventral plaque located below the middle of scape; scape length/width 3.3; lengths scape/head (dorsal view) 0.5; scape length/HE 0.9; length/width F1, F2, F3, F4, clava: 2.3, 2.5, 2.3, 3.0, 7.8; length dorsobasal setae on F1/length F1 0.6. Mesosoma. Length/width 1.4; mesoscutum length/width 0.6; mesoscutellum length/width 0.7; widths SMG/ SLG 1.4; enclosed space between SMG length/width 1.6; lengths mesoscutum/mesoscutellum 1.6; lengths mesoscutellum/dorsellum 2.6; lengths mesosoma/gaster 0.8. Wings. CC length/width 18.8; lengths CC/MV 0.9; lengths MV/ST 2.0; lengths MV/ PM 4.0; lengths PM/ST 0.5; submarginal vein with four setae (four setae in paratypes) on dorsal surface. Gaster. With lateral tufts of pale and flattened setae on $\mathrm{Gt}_{6}$.

Etymology. Named after John S. Noyes (NHMUK), collector of the material of this and several other species described here.

\section{Eriastichus maniatis sp. nov.}

http://zoobank.org/B3BA25D5-CA2F-4B3D-AB17-B8AAF4848F7D

Figure 36

Type locality. Costa Rica, Puntarenas, San Vito, Las Cruces, $8^{\circ} 46^{\prime} \mathrm{N}, 82^{\circ} 57^{\prime} \mathrm{W}$, 1300 m, 15-16.ii.2006, J.S. Noyes leg.

Type specimen. Holotype male dried and glued to a paper card. Original labels: "COSTA RICA, Puntarenas, San Vito, Las Cruces, $8^{\circ} 46^{\prime} \mathrm{N}, 82^{\circ} 57^{\prime} \mathrm{W}, 1300$ m, 15-16. ii.2006, J.S. Noyes", "HOLOTYPE Eriastichus maniatis Hansson” [red printed label], (NHMUK014431052). 
Diagnosis (male). Head dark brown with parts above mouth yellowish brown, scape yellowish brown, pedicel and flagellum brown; ventral plaque on scape ca. 0.3 $x$ as long as scape (Fig. 36), antenna with dorsobasal setae on F1 $0.6 \times$ as long as F1; gaster with lateral tufts of pale and flattened setae on $\mathrm{Gt}_{6}$.

Description (male holotype NHMUK014431052). Length of body $1.5 \mathrm{~mm}$. Head dark brown with parts above mouth yellowish brown, scape yellowish brown, pedicel and flagellum brown, ventral plaque dark brown. Mesoscutum and mesoscutellum black with metallic tinges, dorsellum and propodeum dark brown. Legs yellowish brown. Gaster dark brown.

Head. Length/width in frontal view 0.8 ; width/length in dorsal view 2.2; POL/ OOL 2.1; WM/MS 1.4; MS/HE 0.5; HE/head length in frontal view 0.6; widths head/mesoscutum 1.3. Antenna. Pedicel + flagellum length/mesoscutum width 2.6; pedicel + flagellum length/head width 2.1; lengths scape/ventral plaque 3.8; ventral plaque located below the middle of scape; scape length/width 3.8; lengths scape/head (dorsal view) 0.5; scape length/HE 0.9; length/width F1, F2, F3, F4, clava: 3.7, 3.8, 3.8, 3.7, 8.3; length dorsobasal setae on F1/length F1 0.6. Mesosoma. Length/width 1.6; mesoscutum length/width 0.5 ; mesoscutellum length/ width 0.8; widths SMG/SLG 1.0; enclosed space between SMG length/width 2.3; lengths mesoscutum/mesoscutellum 1.1; lengths mesoscutellum/dorsellum 3.1; lengths mesosoma/gaster 0.9. Wings. CC length/width 21.7; lengths CC/MV 0.9; lengths MV/ST 2.5; lengths MV/PM 3.4; lengths PM/ST 0.7; submarginal vein with seven setae on dorsal surface. Gaster. With lateral tufts of pale and flattened setae on $\mathrm{Gt}_{6}$.

\section{Eriastichus masneri La Salle}

Eriastichus masneri La Salle, 1994: 207. Holotype female in CNC, not examined.

Diagnosis. Mesoscutellum with parts lateral to submedian grooves strongly setose (as in Fig. 6); median propodeum hairy (fig. 30 in La Salle (1994)).

Description. See La Salle (1994).

Distribution. Dominican Republic (La Salle 1994).

\section{Eriastichus nakos La Salle}

Eriastichus nakos La Salle, 1994: 208. Holotype female in CNC, not examined.

Diagnosis. Mesoscutellum with parts lateral to submedian grooves strongly setose (as in Fig. 6); median propodeum bare (as in Fig. 5).

Description. See La Salle (1994).

Distribution. Dominican Republic, Ecuador (La Salle 1994). 


\section{Eriastichus nebulis sp. nov.}

http://zoobank.org/5759023B-4DDD-48BC-BC00-CF7B4ACC38F4

Figure 38

Type locality. Costa Rica, Puntarenas, San Vito, Estación Biológica Las Alturas, $1500 \mathrm{~m}, 8^{\circ} 57^{\prime} \mathrm{N}, 82^{\circ} 50^{\prime} \mathrm{W}, 17-18 . i i .2012$, J.S. Noyes leg.

Type specimen. Holotype male dried and glued to a paper card. Original labels: "COSTA RICA, Puntarenas, San Vito, E.B. Las Alturas, $1500 \mathrm{~m}, 8^{\circ} 57^{\prime} \mathrm{N}, 82^{\circ} 50^{\prime} \mathrm{W}$, 17-18.ii.2012, J.S. Noyes, NHM (Ent) 2012-91", "HOLOTYPE Eriastichus nebulis Hansson" [red printed label], (NHMUK014431053).

Diagnosis (male). Head dark brown with metallic tinges, scape yellowish brown, pedicel and flagellum brown; ventral plaque on scape ca. $0.3 \times$ as long as scape (Fig. 38), antenna with dorsobasal setae on F1 $0.6 \times$ as long as F1; gaster with lateral tufts of pale and flattened setae on $\mathrm{Gt}_{6}$.

Description (male holotype NHMUK014431053). Length of body $1.9 \mathrm{~mm}$. Head dark brown with metallic tinges, scape yellowish brown, pedicel and flagellum brown, ventral plaque dark brown. Mesoscutum and mesoscutellum black with metallic tinges, dorsellum and propodeum dark brown. Legs yellowish brown. Gaster dark brown.

Head. Length/width in frontal view 0.7; width/length in dorsal view 2.2; POL/ OOL 2.3; WM/MS 1.6; MS/HE 0.5; HE/head length in frontal view 0.6; widths head/mesoscutum 1.2. Antenna. Pedicel + flagellum length/mesoscutum width 2.3; pedicel + flagellum length/head width 1.9; lengths scape/ventral plaque 3.0; ventral plaque located below the middle of scape; scape length/width 3.0; lengths scape/head (dorsal view) 0.5; scape length/HE 0.9; length/width F1, F2, F3, F4, clava: 3.6, 3.8, 3.3, 3.3, 7.8; length dorsobasal setae on F1/length F1 0.6. Mesosoma. Length/width 1.7; mesoscutum length/width 0.7; mesoscutellum length/width 0.8; widths SMG/ SLG 1.2; enclosed space between SMG length/width 2.2; lengths mesoscutum/mesoscutellum 1.5; lengths mesoscutellum/dorsellum 2.9; lengths mesosoma/gaster 0.7. Wings. CC length/width 20.0; lengths CC/MV 1.0; lengths MV/ST 2.0; lengths MV/ PM 3.8; lengths PM/ST 0.5; submarginal vein with eight setae on dorsal surface. Gaster. With lateral tufts of pale and flattened setae on $\mathrm{Gt}_{6}$.

\section{Eriastichus neonis sp. nov.}

http://zoobank.org/2D31B245-9233-4289-AD36-D0111F016CC2

Figure 39

Type locality. Costa Rica, Cartago, Humo, El Copal, 947'N, $83^{\circ} 45^{\prime} \mathrm{W}, 1050$ 1250 m, 29.ii-6.iii.2008, C. Hansson leg.

Type specimen. Holotype male dried and glued to a paper card. Original labels: "COSTA RICA, Cartago, Humo, El Copal, 9 $47^{\prime} \mathrm{N}, 83^{\circ} 45^{\prime} \mathrm{W}, 1050-1250$ m, 29.ii6.iii.2008, C. Hansson", "HOLOTYPE Eriastichus neonis Hansson" [red printed label], (MZLU:7040.1). 
Additional type material. Paratypes $(3 \circlearrowleft \hat{\jmath})$ : $1 \widehat{\jmath}$ with same label data as holotype (MZLU:7040.2); 1ठ "COSTA RICA, Heredia, E.B. La Selva, 75 m, LN 264463/532850, 30-31.iii.2002, swept, J. Azofeifa” (MZUCR:01694); 1ठ “COSTA RICA, Puntarenas, San Vito, E.B. Las Alturas, 1500 m, 857'N, 82 $50^{\prime} \mathrm{W}, 17-18$. ii.2012, J.S. Noyes, NHM (Ent) 2012-91” (NHMUK014431054).

Diagnosis (male). Head dark brown, scape yellowish brown, pedicel and flagellum brown; ventral plaque on scape ca. $0.3 \times$ as long as scape (Fig. 39), antenna with dorsobasal setae on F1 $0.7 \times$ as long as F1; gaster with lateral tufts of pale and flattened setae on $\mathrm{Gt}_{6}$.

Description (male holotype MZLU:7040.1). Length of body $1.5 \mathrm{~mm}$ (paratypes 1.3-1.6 mm). Head dark brown, scape yellowish brown, pedicel and flagellum brown, ventral plaque dark brown. Mesoscutum, mesoscutellum and propodeum black, dorsellum dark brown. Legs yellowish brown. Gaster dark brown.

Head. Length/width in frontal view 0.8; width/length in dorsal view 1.9; POL/ OOL 2.5; WM/MS 1.6; MS/HE 0.5; HE/head length in frontal view 0.6; widths head/mesoscutum 1.2. Antenna. Pedicel + flagellum length/mesoscutum width 2.5; pedicel + flagellum length/head width 2.1 ; lengths scape/ventral plaque 4.0 ; ventral plaque located below the middle of scape; scape length/width 3.0; lengths scape/head (dorsal view) 0.5; scape length/HE 0.8; length/width F1, F2, F3, F4, clava: 3.0, 3.0, 3.0, 3.0, 7.0; length dorsobasal setae on F1/length F1 0.7. Mesosoma. Length/width 1.8; mesoscutum length/width 0.7; mesoscutellum length/width 0.9; widths SMG/ SLG 1.2; enclosed space between SMG length/width 2.1; lengths mesoscutum/mesoscutellum 1.5; lengths mesoscutellum/dorsellum 2.6; lengths mesosoma/gaster 0.8 . Wings. CC length/width 23.0; lengths CC/MV 0.9; lengths MV/ST 2.1; lengths MV/ PM 5.0; lengths PM/ST 0.4; submarginal vein with six setae on dorsal surface (6-7 setae in paratypes). Gaster. With lateral tufts of pale and flattened setae on $\mathrm{Gt}_{6}$.

\section{Eriastichus nexilis sp. nov.}

http://zoobank.org/A8B195EF-FC82-4D9C-86DF-DA156C09D23F

Figure 41

Type locality. Costa Rica, Heredia, Estación Biológica La Selva, 75 m, 10²6'N, $84^{\circ} 01^{\prime} \mathrm{W}, 28-29$. ii.2008, J.S. Noyes leg.

Type specimen. Holotype male dried and glued to a paper card. Original labels: "COSTA RICA, Heredia, E.B. La Selva, 75 m, 10²6'N, 8401'W, 28-29.ii.2008, J.S. Noyes, NHM (Ent) 2010-21”, "HOLOTYPE Eriastichus nexilis Hansson" [red printed label], (NHMUK014431055).

Additional type material. Paratype $1 \delta$ “COSTA RICA, Cartago, Humo, El Copal, 947'N, 8345'W, 1050-1250 m, 29.ii-6.iii.2008, C. Hansson” (MZLU:7041.2).

Diagnosis (male). Head dark brown, scape pale brown, pedicel and flagellum brown; ventral plaque on scape ca. $0.2 \times$ as long as scape (Fig. 41), antenna with dorsobasal setae on F1 $0.9 \times$ as long as F1; gaster with lateral tufts of pale and flattened setae on $\mathrm{Gt}_{6}$.

Description (male holotype NHMUK014431055). Length of body $1.1 \mathrm{~mm}$ (paratype $1.2 \mathrm{~mm}$ ). Head dark brown, scape pale brown, pedicel and flagellum brown, 
ventral plaque dark brown. Mesoscutum and mesoscutellum black with metallic tinges, dorsellum dark brown, propodeum black. Legs yellowish brown. Gaster dark brown.

Head. Length/width in frontal view 0.8; width/length in dorsal view 2.2; POL/ OOL 2.0; WM/MS 1.6; MS/HE 0.5; HE/head length in frontal view 0.6; widths head/mesoscutum 1.1. Antenna. Pedicel + flagellum length/mesoscutum width 2.3; pedicel + flagellum length/head width 1.9; lengths scape/ventral plaque 4.2; ventral plaque located below the middle of scape; scape length/width 3.0; lengths scape/head (dorsal view) 0.5; scape length/HE 0.9; length/width F1, F2, F3, F4, clava: 3.3, 3.8, 3.5, 3.5, 8.5; length dorsobasal setae on F1/length F1 0.9. Mesosoma. Length/width 1.4; mesoscutum length/width 0.6; mesoscutellum length/width 0.8; widths SMG/ SLG 1.1; enclosed space between SMG length/width 2.1; lengths mesoscutum/mesoscutellum 1.4; lengths mesoscutellum/dorsellum 3.2; lengths mesosoma/gaster 0.7. Wings. CC length/width 22.5; lengths CC/MV 1.1; lengths MV/ST 1.9; lengths MV/ PM 4.0; lengths PM/ST 0.5; submarginal vein with six setae on dorsal surface (five setae in paratype). Gaster. With lateral tufts of pale and flattened setae on $\mathrm{Gt}_{6}$.

\section{Eriastichus novalis sp. nov.}

http://zoobank.org/A656E7CC-7D18-4A45-BDB8-F5F05D7C539D

Figure 44

Type locality. Costa Rica, San José, San Gerardo de Dota, 9³3'N, 8347'W, 20-21. ii.2013, J.S. Noyes leg.

Type specimen. Holotype male dried and glued to a paper card. Original labels: "COSTA RICA, San José, San Gerardo de Dota, 20-21.ii.2013, J.S. Noyes, NHM (Ent) 2012-91", "HOLOTYPE Eriastichus novalis Hansson" [red printed label], (NHMUK014431056).

Diagnosis (male). Head dark brown with part below antennal toruli yellowish brown, scape yellowish brown, pedicel pale brown, flagellum brown; ventral plaque on scape ca. $0.4 \times$ as long as scape (Fig. 44), antenna with dorsobasal setae on F1 $0.9 \times$ as long as F1; gaster with lateral tufts of pale and flattened setae on $\mathrm{Gt}_{6}$.

Description (male holotype NHMUK014431056). Length of body $1.7 \mathrm{~mm}$. Head dark brown with part below antennal toruli yellowish brown, scape yellowish brown, pedicel pale brown, flagellum brown, ventral plaque dark brown. Mesoscutum and mesoscutellum dark brown with metallic tinges, dorsellum pale brown, propodeum dark brown. Legs yellowish brown. Gaster dark brown.

Head. Length/width in frontal view 0.7 ; width/length in dorsal view 2.3; POL/ OOL 1.8; WM/MS 1.6; MS/HE 0.5; HE/head length in frontal view 0.6; widths head/mesoscutum 1.0. Antenna. Pedicel + flagellum length/mesoscutum width 2.0; pedicel + flagellum length/head width 1.8 ; lengths scape/ventral plaque 2.5 ; ventral plaque located below the middle of scape; scape length/width 2.5; lengths scape/head (dorsal view) 0.5; scape length/HE 0.8; length/width F1, F2, F3, F4, clava: 3.2, 3.0, 2.7, 2.8, 6.5; length dorsobasal setae on F1/length F1 0.9. Mesosoma. Length/width 1.4; mesoscutum length/width 0.6; mesoscutellum length/width 1.9; widths SMG/ 


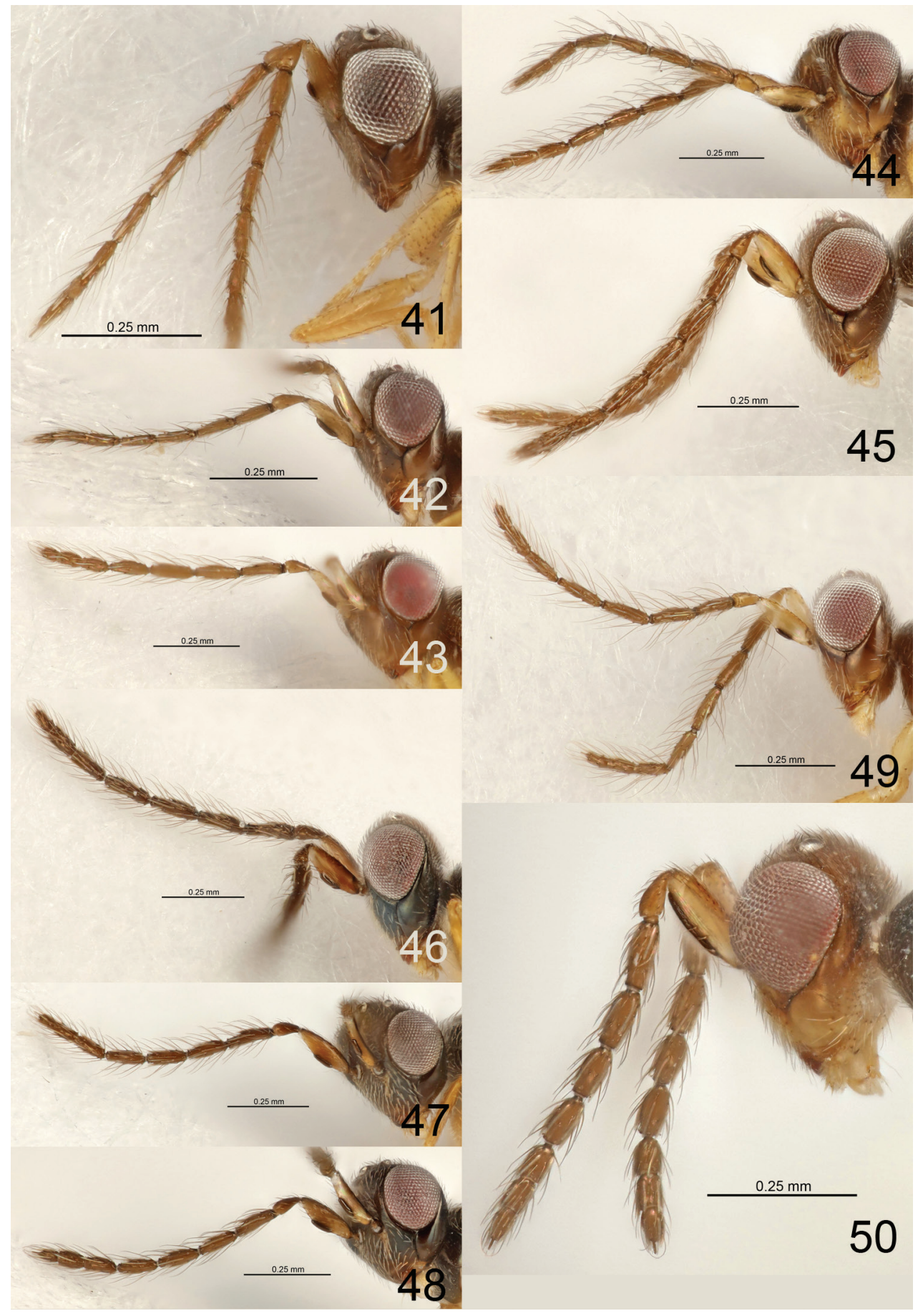

Figures 4I-50. Eriastichus spp. head and antenna in lateral view, male holotypes 4 I E. nexilis $\mathbf{4 2} E$. oasis 43 E. ononis 44 E. novalis 45 E. nugalis 46 E. parabilis 47 E. rivalis 48 E. sodalis 49 E. orestis 50 E. pallidops. 
SLG 1.0; enclosed space between SMG length/width 2.0; lengths mesoscutum/mesoscutellum 1.6; lengths mesoscutellum/dorsellum 2.6; lengths mesosoma/gaster 0.7. Wings. CC length/width 23.3; lengths CC/MV 1.0; lengths MV/ST 2.1; lengths MV/PM 5.4; lengths PM/ST 0.4; submarginal vein with ten setae on dorsal surface.

Gaster. With lateral tufts of pale and flattened setae on $\mathrm{Gt}_{6}$.

\section{Eriastichus nugalis sp. nov.}

http://zoobank.org/07A69A24-BEB7-4C6C-BA98-953B99362A62

Figure 45

Type locality. Costa Rica, Cartago, Humo, El Copal, 947'N, 8345'W, 1050 1250 m, 29.ii-6.iii.2008, C. Hansson leg.

Type specimen. Holotype male dried and glued to a paper card. Original labels: "COSTA RICA, Cartago, Humo, El Copal, 947'N, 8345'W, 1050-1250 m, 29.ii6.iii.2008, C. Hansson", "HOLOTYPE Eriastichus nugalis Hansson" [red printed label], (MZLU:7042.1).

Additional type material. Paratypes $(2 \lesssim \widehat{\circlearrowleft}): 1 \hat{\jmath}$ with same label data as holotype (MZLU:7042.2); $1 \sigma^{\Uparrow}$ "COSTA RICA, Puntarenas, San Vito, E.B. Las Alturas, $1500 \mathrm{~m}, 8^{\circ} 57^{\prime} \mathrm{N}, 82^{\circ} 50^{\prime} \mathrm{W}, 17-18 . i i .2012$, J.S. Noyes, NHM (Ent) 2012-91” (NHMUK014431057).

Diagnosis (male). Head dark brown, scape yellowish brown, pedicel pale brown, flagellum dark brown; ventral plaque on scape ca. $0.4 \times$ as long as scape (Fig. 45), antenna with dorsobasal setae on F1 $0.5 \times$ as long as F1; gaster with lateral tufts of pale and flattened setae on $\mathrm{Gt}_{6}$.

Description (male holotype MZLU:7042.1). Length of body $1.8 \mathrm{~mm}$ (paratypes 1.5-1.6 mm). Head dark brown, scape yellowish brown, pedicel pale brown, flagellum dark brown, ventral plaque dark brown. Mesoscutum, mesoscutellum and propodeum dark brown, dorsellum pale brown. Legs with fore and mid coxae yellowish brown with base brown, hind coxa, remaining parts of legs yellowish brown. Gaster dark brown.

Head. Length/width in frontal view 0.8; width/length in dorsal view 2.1; POL/ OOL 1.8; WM/MS 1.9; MS/HE 0.5; HE/head length in frontal view 0.6; widths head/mesoscutum 1.1. Antenna. Pedicel + flagellum length/mesoscutum width 2.1; pedicel + flagellum length/head width 1.9; lengths scape/ventral plaque 2.5; ventral plaque located in the middle of scape; scape length/width 3.0; lengths scape/head (dorsal view) 0.6; scape length/HE 0.9; length/width F1, F2, F3, F4, clava: 2.5, 2.5, 2.5, 2.5, 5.5; length dorsobasal setae on F1/length F1 0.5. Mesosoma. Length/width 1.6; mesoscutum length/width 0.6; mesoscutellum length/width 0.8 ; widths SMG/SLG 1.0; enclosed space between SMG length/width 2.3; lengths mesoscutum/mesoscutellum 1.4; lengths mesoscutellum/dorsellum 2.8; lengths mesosoma/gaster 0.7. Wings. CC length/width 30.0; lengths CC/MV 1.1; lengths MV/ST 1.9; lengths MV/PM 4.9; lengths PM/ST 0.4; submarginal vein with eight setae on dorsal surface (paratypes with seven setae). Gaster. With lateral tufts of pale and flattened setae on $\mathrm{Gt}_{6}$. 


\section{Eriastichus oasis sp. nov.}

http://zoobank.org/3008B625-977E-4985-90B1-81A4783D1BB3

Figure 42

Type locality. Costa Rica, Heredia, Estación Biológica La Selva, 75 m, 10²6'N, $84^{\circ} 01^{\prime} \mathrm{W}, 22-24 . i i .2012$, J.S. Noyes leg.

Type specimen. Holotype male dried and glued to a paper card. Original labels: "COSTA RICA, Heredia, E.B. La Selva, 75 m, 10²6'N, 8401 'W, 22-24.ii.2012, J.S. Noyes, NHM (Ent) 2012-91", "HOLOTYPE Eriastichus oasis Hansson" [red printed label], (NHMUK014431058).

Additional type material. Paratype 10 "COSTA RICA, Cartago, Humo, El Copal, $9^{\circ} 47^{\prime} \mathrm{N}, 83^{\circ} 45^{\prime} \mathrm{W}, 1050-1250 \mathrm{~m}, 29$. .ii-6.iii.2008, C. Hansson” (MZLU:7043.2).

Diagnosis (male). Head dark brown, scape yellowish brown, pedicel pale brown, flagellum brown; ventral plaque on scape ca. $0.5 \times$ as long as scape (Fig. 42), antenna with dorsobasal setae on F1 $0.5 \times$ as long as F1; gaster with lateral tufts of pale and flattened setae on $\mathrm{Gt}_{6}$.

Description (male holotype NHMUK014431058). Length of body $1.3 \mathrm{~mm}$ (paratype $1.4 \mathrm{~mm}$ ). Head dark brown, scape yellowish brown, pedicel pale brown, flagellum brown, ventral plaque dark brown. Mesoscutum and mesoscutellum black with metallic tinges, dorsellum pale brown, propodeum dark brown. Legs yellowish brown, hind coxa with base dark brown. Gaster dark brown.

Head. Length/width in frontal view 0.8 ; width/length in dorsal view 2.3; POL/ OOL 2.0; WM/MS 1.5; MS/HE 0.5; HE/head length in frontal view 0.6; widths head/mesoscutum 1.2. Antenna. Pedicel + flagellum length/mesoscutum width 2.1; pedicel + flagellum length/head width 1.9; lengths scape/ventral plaque 2.2; ventral plaque located below the middle of scape; scape length/width 3.1; lengths scape/head (dorsal view) 0.5; scape length/HE 0.8; length/width F1, F2, F3, F4, clava: 3.3, 3.3, 3.3, 3.0, 8.5; length dorsobasal setae on F1/length F1 0.5. Mesosoma. Length/width 1.5; mesoscutum length/width 0.5; mesoscutellum length/width 0.6; widths SMG/ SLG 0.9; enclosed space between SMG length/width 2.0; lengths mesoscutum/mesoscutellum 1.2; lengths mesoscutellum/dorsellum 2.3; lengths mesosoma/gaster 0.7. Wings. CC length/width 19.0; lengths CC/MV 1.2; lengths MV/ST 2.1; lengths MV/ PM 4.1; lengths PM/ST 0.5; submarginal vein with seven setae on dorsal surface (six setae in paratype). Gaster. With lateral tufts of pale and flattened setae on $\mathrm{Gt}_{6}$.

\section{Eriastichus ononis sp. nov.}

http://zoobank.org/A538731B-FECE-4287-9566-65DEC5E3F735

Figure 43

Type locality. Costa Rica, Puntarenas, San Vito, Las Cruces, $8^{\circ} 46^{\prime} \mathrm{N}, 82^{\circ} 57^{\prime} \mathrm{W}$, 1300 m, 15-16.ii.2006, J.S. Noyes leg. 
Type specimen. Holotype male dried and glued to a paper card. Original labels: "COSTA RICA, Puntarenas, San Vito, Las Cruces, 846'N, 8257'W, 1300 m, 15-16. ii.2006, J.S. Noyes", "HOLOTYPE Eriastichus ononis Hansson" [red printed label], (NHMUK014431059).

Diagnosis (male). Head dark brown, scape yellowish brown, pedicel and flagellum brown; ventral plaque on scape ca. $0.3 \times$ as long as scape (Fig. 43), antenna with dorsobasal setae on F1 $0.9 \times$ as long as F1; gaster with lateral tufts of pale and flattened setae on $\mathrm{Gt}_{6}$.

Description (male holotype NHMUK014431059). Length of body $1.5 \mathrm{~mm}$. Head dark brown, scape yellowish brown, pedicel and flagellum brown, ventral plaque dark brown. Mesoscutum and mesoscutellum black with metallic tinges, dorsellum pale brown, propodeum dark brown. Legs yellowish brown. Gaster dark brown.

Head. Length/width in frontal view 0.8 ; width/length in dorsal view 2.1; POL/ OOL 2.4; WM/MS 1.5; MS/HE 0.5; HE/head length in frontal view 0.6; widths head/mesoscutum 1.2. Antenna. Pedicel + flagellum length/mesoscutum width 2.5; pedicel + flagellum length/head width 2.0; lengths scape/ventral plaque 3.1; ventral plaque located below the middle of scape; scape length/width 2.8; lengths scape/head (dorsal view) 0.5; scape length/HE 0.9; length/width F1, F2, F3, F4, clava: 3.6, 3.6, 3.6, 3.4, 7.4; length dorsobasal setae on F1/length F1 0.9. Mesosoma. Length/width 1.7; mesoscutum length/width 0.7; mesoscutellum length/width 0.9; widths SMG/ SLG 1.0; enclosed space between SMG length/width 2.7; lengths mesoscutum/mesoscutellum 1.4; lengths mesoscutellum/dorsellum 3.4; lengths mesosoma/gaster 0.7. Wings. CC length/width 16.0; lengths CC/MV 1.0; lengths MV/ST 2.1; lengths MV/ PM 5.0; lengths PM/ST 0.4; submarginal vein with seven setae on dorsal surface. Gaster. With lateral tufts of pale and flattened setae on $\mathrm{Gt}_{6}$.

\section{Eriastichus orestis sp. nov.}

http://zoobank.org/C962EAC3-7329-4811-A87E-EE829A92C069

Figure 49

Type locality. Costa Rica, Puntarenas, San Vito, Estación Biológica Las Alturas, $1500 \mathrm{~m}, 8^{\circ} 57^{\prime} \mathrm{N}, 82^{\circ} 50^{\prime} \mathrm{W}, 17-18 . i i .2012$, J.S. Noyes leg.

Type specimen. Holotype male dried and glued to a paper card. Original labels: "COSTA RICA, Puntarenas, San Vito, E.B. Las Alturas, 1500 m, 8 $57^{\prime} \mathrm{N}, 82^{\circ} 50^{\prime} \mathrm{W}$, 17-18.ii.2012, J.S. Noyes, NHM (Ent) 2012-91", "HOLOTYPE Eriastichus orestis Hansson" [red printed label], (NHMUK014431060).

Additional type material. Paratypes $(2 \hat{\partial} \hat{\jmath}): 1 \hat{\jmath}$ with same label data as holotype (NHMUK014431061); $10^{\lambda}$ “COSTA RICA, Cartago, Humo, El Copal, 947'N, 8345'W, 1050-1250 m, 29.ii-6.iii.2008, C. Hansson” (MZLU:7044.2).

Diagnosis (male). Head dark brown with part below antennal toruli dark yellowish brown, scape yellowish brown, pedicel and flagellum brown; ventral plaque on 
scape ca. $0.3 \times$ as long as scape (Fig. 49), antenna with dorsobasal setae on F1 $0.9 \times$ as long as F1; gaster with lateral tufts of pale and flattened setae on $\mathrm{Gt}_{6}$.

Description (male holotype NHMUK014431060). Length of body $1.7 \mathrm{~mm}$ (paratypes 1.7-1.8 mm). Head dark brown with part below antennal toruli pale brown, scape yellowish brown, pedicel pale brown, flagellum brown, ventral plaque dark brown. Mesoscutum, mesoscutellum and propodeum dark brown, dorsellum pale brown. Legs yellowish brown. Gaster dark brown.

Head. Length/width in frontal view 0.7 ; width/length in dorsal view 2.3; POL/ OOL 1.8; WM/MS 1.5; MS/HE 0.6; HE/head length in frontal view 0.6; widths head/mesoscutum 1.3. Antenna. Pedicel + flagellum length/mesoscutum width 2.3; pedicel + flagellum length/head width 1.9; lengths scape/ventral plaque 3.0; ventral plaque located below the middle of scape; scape length/width 3.0; lengths scape/head (dorsal view) 0.5; scape length/HE 0.9; length/width F1, F2, F3, F4, clava: 2.8, 3.0, 3.2, 3.2, 6.8; length dorsobasal setae on F1/length F1 0.9. Mesosoma. Length/width 1.6; mesoscutum length/width 0.7; mesoscutellum length/width 0.8; widths SMG/ SLG 1.1; enclosed space between SMG length/width 2.4; lengths mesoscutum/mesoscutellum 1.5; lengths mesoscutellum/dorsellum 3.3; lengths mesosoma/gaster 0.7. Wings. CC length/width 16.2; lengths CC/MV 0.7; lengths MV/ST 2.4; lengths MV/ PM 4.8; lengths PM/ST 0.5; submarginal vein with six setae on dorsal surface (paratypes with eight setae). Gaster. With lateral tufts of pale and flattened setae on $\mathrm{Gt}_{6}$.

\section{Eriastichus pallidops sp. nov.}

http://zoobank.org/A4B4B83F-D622-49B8-965F-CECE0B604217

Figures 50, 59

Type locality. Costa Rica, Cartago, Humo, El Copal, 947'N, $83^{\circ} 45^{\prime} \mathrm{W}, 1050$ 1250 m, 29.ii-6.iii.2008, C. Hansson leg.

Type specimen. Holotype male dried and glued to a paper card. Original labels: "COSTA RICA, Cartago, Humo, El Copal, $9^{\circ} 47^{\prime} \mathrm{N}, 83^{\circ} 45^{\prime} \mathrm{W}, 1050-1250$ m, 29.ii6.iii.2008, C. Hansson", "HOLOTYPE Eriastichus pallidops Hansson" [red printed label], (MZLU:7045.1).

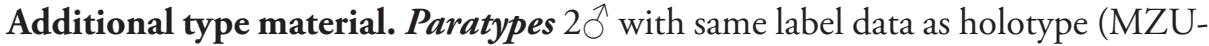
CR:01695, NHMUK014431062).

Diagnosis (male). Head with lower $1 / 2$ yellowish brown (Fig. 59); antenna (Fig. 50): scape yellowish brown, ventral plaque on scape $0.7 \times$ as long as scape, antenna with dorsobasal setae on F1 $0.4 \times$ as long as F1; gaster with lateral tufts of pale and flattened setae on $\mathrm{Gt}_{5-6}$.

Description (male holotype MZLU:7045.1). Length of body $1.9 \mathrm{~mm}$ (paratypes $1.5-1.7 \mathrm{~mm}$ ). Head yellowish brown with upper $1 / 2$ of frons pale brown, vertex and upper $1 / 2$ of occiput dark brown. Antenna with scape yellowish brown with dorsal margin pale brown, ventral plaque black; pedicel and flagellum brown. Mesoscutum and mesoscutellum black with metallic tinges; dorsellum pale brown; propodeum dark 
brown. Legs yellowish brown with base of hind coxa and $4^{\text {th }}$ tarsomere dark brown. Gaster dark brown.

Head. Length/width in frontal view 0.7; width/length in dorsal view 2.2; POL/ OOL 1.8; WM/MS 1.7; MS/HE 0.5; HE/head length in frontal view 0.6; widths head/ mesoscutum 1.1. Antenna. Pedicel + flagellum length/mesoscutum width 1.5; pedicel + flagellum length/head width 1.4; lengths scape/ventral plaque 1.4; ventral plaque located slightly above the middle of scape; scape length/width 2.8; lengths scape/head (dorsal view) 0.6; scape length/HE 0.9; length/width F1, F2, F3, F4, clava: 2.3, 2.0, 1.8, 1.8, 4.0; length dorsobasal setae on F1/length F1 0.4. Mesosoma. Length/width 1.6; mesoscutum length/width 0.5; mesoscutellum length/width 0.7; widths SMG/ SLG 1.2; enclosed space between SMG length/width 1.8; lengths mesoscutum/mesoscutellum 1.2; lengths mesoscutellum/dorsellum 2.7; lengths mesosoma/gaster 0.8 . Wings. CC length/width 19.3; lengths CC/MV 1.1; lengths MV/ST 2.2; lengths MV/ PM 3.7; lengths PM/ST 0.6; submarginal vein with ten setae on dorsal surface (8-9 setae in paratypes). Gaster. With lateral tufts of pale and flattened setae on $\mathrm{Gt}_{5-6}$.

Etymology. Named for the yellowish brown lower face.

\section{Eriastichus parabilis sp. nov.}

http://zoobank.org/F33852D4-65AF-4B99-9A79-A5C468443274

Figure 46

Type locality. Costa Rica, San José, San Gerardo de Dota, 9³3'N, 8347'W, 20-21. ii.2013, J.S. Noyes leg.

Type specimen. Holotype male dried and glued to a paper card. Original labels: "COSTA RICA, San José, San Gerardo de Dota, 20-21.ii.2013, J.S. Noyes, NHM (Ent) 2012-91", "HOLOTYPE Eriastichus parabilis Hansson" [red printed label], (NHMUK014431063).

Additional type material. Paratype $1 \hat{\jmath}$ with same label data as holotype (NHMUK014431064).

Diagnosis (male). Head black with lower part of clypeus yellowish brown, scape pale brown, pedicel and flagellum dark brown; ventral plaque on scape ca. $0.4 \times$ as long as scape (Fig. 46), antenna with dorsobasal setae on F1 $0.5 \times$ as long as F1; gaster with lateral tufts of pale and flattened setae on $\mathrm{Gt}_{6}$.

Description (male holotype NHMUK014431063). Length of body $2.3 \mathrm{~mm}$ (paratype $2.3 \mathrm{~mm}$ ). Head black with lower part of clypeus yellowish brown, scape pale brown, pedicel and flagellum dark brown, ventral plaque dark brown. Mesoscutum, mesoscutellum, dorsellum and propodeum black with metallic tinges. Legs with hind coxa black, hind femur pale brown, remaining parts of legs yellowish brown. Wings uniformly but weakly infuscated. Gaster dark brown.

Head. Length/width in frontal view 0.7; width/length in dorsal view 2.3; POL/ OOL 1.9; WM/MS 1.5; MS/HE 0.6; HE/head length in frontal view 0.6; widths head/mesoscutum 1.1. Antenna. Pedicel + flagellum length/mesoscutum width 2.2; 
pedicel + flagellum length/head width 2.0; lengths scape/ventral plaque 2.8; ventral plaque located below the middle of scape; scape length/width 3.1; lengths scape/head (dorsal view) 0.7; scape length/HE 0.9; length/width F1, F2, F3, F4, clava: 3.1, 3.0, 2.9, 2.9, 6.3; length dorsobasal setae on F1/length F1 0.5. Mesosoma. Length/width 1.7; mesoscutum length/width 0.7; mesoscutellum length/width 0.8 ; widths SMG/ SLG 1.3; enclosed space between SMG length/width 2.1; lengths mesoscutum/mesoscutellum 1.5; lengths mesoscutellum/dorsellum 3.2; lengths mesosoma/gaster 0.8 . Wings. CC length/width 17.5; lengths CC/MV 1.0; lengths MV/ST 1.9; lengths MV/ PM 5.1; lengths PM/ST 0.4; submarginal vein with ten setae on dorsal surface (nine setae in paratype). Gaster. With lateral tufts of pale and flattened setae on $\mathrm{Gt}_{6}$.

\section{Eriastichus renodis sp. nov.}

http://zoobank.org/1BC71A82-5B85-4E61-BD84-27543158E22C

Figure 54

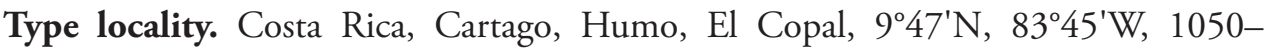
1250 m, 29.ii-6.iii.2008, C. Hansson leg.

Type specimen. Holotype male dried and glued to a paper card. Original labels: "COSTA RICA, Cartago, Humo, El Copal, 9 $47^{\prime} \mathrm{N}, 83^{\circ} 45^{\prime} \mathrm{W}, 1050-1250$ m, 29.ii6.iii.2008, C. Hansson", "HOLOTYPE Eriastichus renodis Hansson" [red printed label], (MZLU:7046.1).

Diagnosis (male). Head dark brown, scape yellowish brown, pedicel pale brown, flagellum brown; ventral plaque on scape ca. $0.3 \times$ as long as scape (Fig. 54), antenna with dorsobasal setae on F1 $0.9 \times$ as long as F1; gaster with lateral tufts of pale and flattened setae on $\mathrm{Gt}_{6}$.

Description (male holotype MZLU:7046.1). Length of body $1.5 \mathrm{~mm}$. Head dark brown, scape yellowish brown, pedicel pale brown, flagellum brown, ventral plaque dark brown. Mesoscutum and mesoscutellum dark brown with metallic tinges, dorsellum and propodeum dark brown. Legs yellowish brown. Gaster dark brown.

Head. Length/width in frontal view 0.7; width/length in dorsal view 2.2; POL/ OOL 2.3; WM/MS 1.3; MS/HE 0.6; HE/head length in frontal view 0.6; widths head/mesoscutum 1.2. Antenna. Pedicel + flagellum length/mesoscutum width 2.4; pedicel + flagellum length/head width 2.0; lengths scape/ventral plaque 3.1; ventral plaque located in the middle of scape; scape length/width 3.1; lengths scape/head (dorsal view) 0.6; scape length/HE 0.9; length/width F1, F2, F3, F4, clava: 3.2, 3.4, 3.4, 3.4, 7.6; length dorsobasal setae on F1/length F1 0.9. Mesosoma. Length/width 1.7; mesoscutum length/width 0.6; mesoscutellum length/width 0.9; widths SMG/SLG 1.1 ; enclosed space between SMG length/width 2.3; lengths mesoscutum/mesoscutellum 1.4; lengths mesoscutellum/dorsellum 2.9; lengths mesosoma/gaster 0.8. Wings. CC length/width 20.0; lengths CC/MV 0.8; lengths MV/ST 2.3; lengths MV/PM 


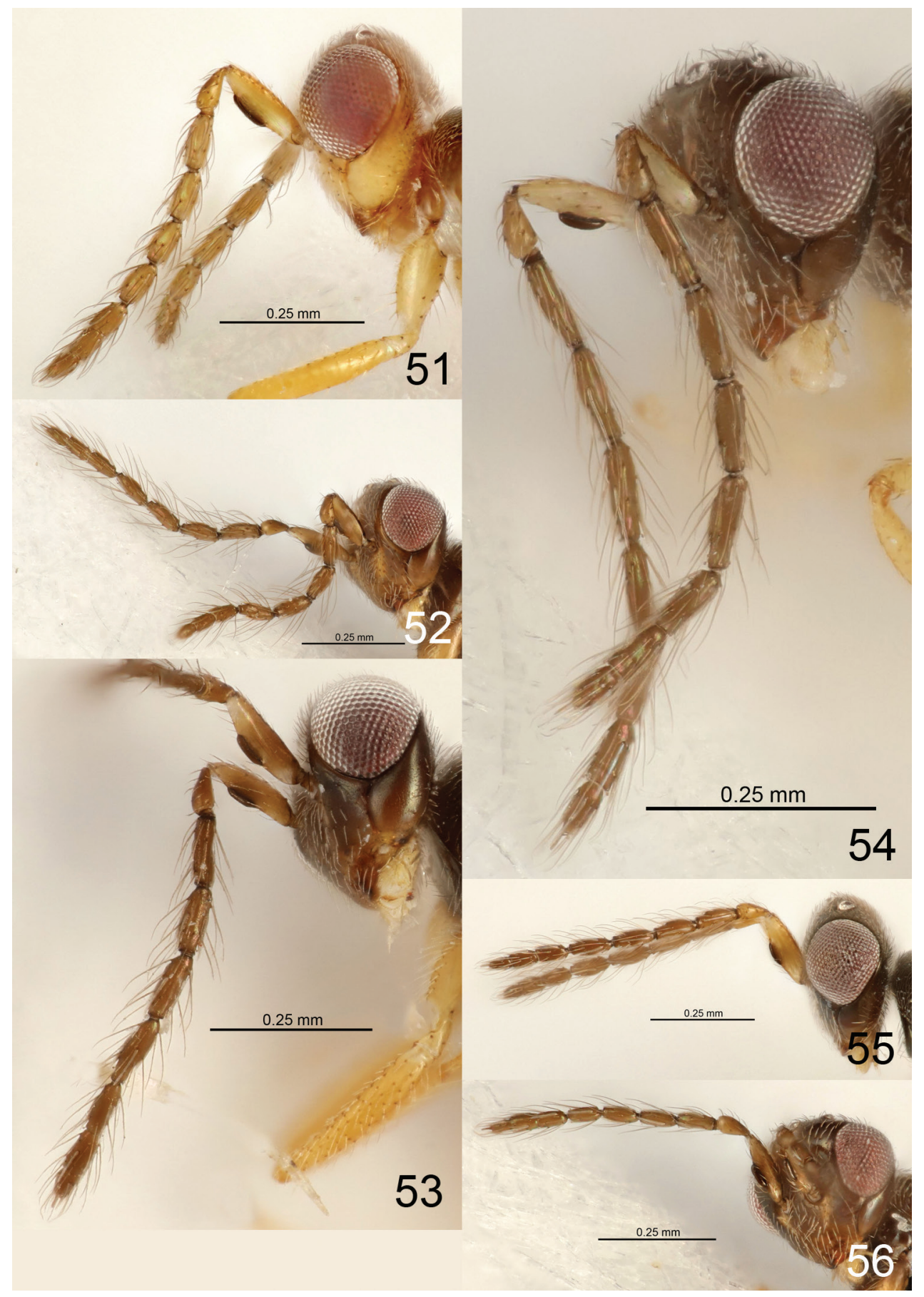

Figures 5|-56. Eriastichus spp. head and antenna in lateral view, male holotypes $\mathbf{5} \mathbf{I}$ E. sannionis $\mathbf{5 2}$ E. scalaris $\mathbf{5 3}$ E. taraxis $\mathbf{5 4}$ E. renodis $\mathbf{5 5}$ E. tendrilis $\mathbf{5 6}$ E. velaminis. 
4.2; lengths PM/ST 0.5; submarginal vein with nine setae on dorsal surface. Gaster. With lateral tufts of pale and flattened setae on $\mathrm{Gt}_{6}$.

\section{Eriastichus rivalis sp. nov.}

http://zoobank.org/F78216A7-C07E-4635-A5E5-921D46D5E6AA

Figure 47

Type locality. Costa Rica, San José, San Gerardo de Dota, 9³3'N, 834ㄱ'W, $20-21$. ii.2013, J.S. Noyes leg.

Type specimen. Holotype male dried and glued to a paper card. Original labels: "COSTA RICA, San José, San Gerardo de Dota, 20-21.ii.2013, J.S. Noyes, NHM (Ent) 2012-91", "HOLOTYPE Eriastichus rivalis Hansson" [red printed label], (NHMUK014431065).

Diagnosis (male). Head black, scape pale brown, pedicel and flagellum dark brown; ventral plaque on scape ca. $0.4 \times$ as long as scape (Fig. 47), antenna with dorsobasal setae on F1 $0.5 \times$ as long as F1; gaster with lateral tufts of pale and flattened setae on $\mathrm{Gt}_{6}$.

Description (male holotype NHMUK014431065). Length of body $2.0 \mathrm{~mm}$. Head black, scape pale brown, pedicel and flagellum dark brown, ventral plaque dark brown. Mesoscutum, mesoscutellum, dorsellum and propodeum black with metallic tinges. Legs with mid and hind coxae dark brown, hind femur pale brown, remaining parts of legs yellowish brown. Gaster dark brown.

Head. Length/width in frontal view 0.8 ; width/length in dorsal view 2.3; POL/ OOL 2.4; WM/MS 1.4; MS/HE 0.6; HE/head length in frontal view 0.6; widths head/mesoscutum 1.2. Antenna. Pedicel + flagellum length/mesoscutum width 2.0; pedicel + flagellum length/head width 1.9; lengths scape/ventral plaque 2.8; ventral plaque located below the middle of scape; scape length/width 3.1; lengths scape/head (dorsal view) 0.6; scape length/HE 1.0; length/width F1, F2, F3, F4, clava: 3.7, 3.2, 3.2, 3.2, 7.3; length dorsobasal setae on F1/length F1 0.5. Mesosoma. Length/width 1.6; mesoscutum length/width 0.6; mesoscutellum length/width 0.7; widths SMG/ SLG 1.2; enclosed space between SMG length/width 2,0; lengths mesoscutum/mesoscutellum 1.5; lengths mesoscutellum/dorsellum 2.7; lengths mesosoma/gaster 0.7. Wings. CC length/width 21.3; lengths CC/MV 1.0; lengths MV/ST 2.2; lengths MV/PM 4.1; lengths PM/ST 0.5; submarginal vein with nine setae on dorsal surface. Gaster. With lateral tufts of pale and flattened setae on $\mathrm{Gt}_{6}$.

\section{Eriastichus sannionis sp. nov.}

http://zoobank.org/F30DC6E4-DDEE-4240-B603-BD32C6EA5DE2

Figure 51

Type locality. Costa Rica, Cartago, Humo, El Copal, 947'N, 83²4'W, 1050 1250 m, 29.ii-6.iii.2008, C. Hansson leg. 
Type specimen. Holotype male dried and glued to a paper card. Original labels: "COSTA RICA, Cartago, Humo, El Copal, 9 $47^{\prime} \mathrm{N}, 83^{\circ} 45^{\prime} \mathrm{W}, 1050-1250$ m, 29.ii6.iii.2008, C. Hansson", "HOLOTYPE Eriastichus sannionis Hansson" [red printed label], (MZLU:7047.1).

Additional type material. Paratype $1 \delta^{\hat{~}}$ with same label data as holotype (NHMUK014431066).

Diagnosis (male). Head yellowish brown with vertex and upper $1 / 2$ of occiput brown, antenna yellowish brown; ventral plaque on scape ca. $0.5 \times$ as long as scape (Fig. 51), antenna with dorsobasal setae on F1 $0.6 \times$ as long as F1; gaster with lateral tufts of pale and flattened setae on $\mathrm{Gt}_{5-6}$.

Description (male holotype MZLU:7047.1). Length of body $1.5 \mathrm{~mm}$ (paratype $1.3 \mathrm{~mm}$ ). Head yellowish brown with vertex and upper $1 / 2$ of occiput brown, antenna yellowish brown, ventral plaque dark brown. Mesoscutum and propodeum dark brown, mesoscutellum black with metallic tinges, dorsellum pale brown. Legs yellowish brown. Gaster dark brown.

Head. Length/width in frontal view 0.7 ; width/length in dorsal view 2.2; POL/ OOL 2.1; WM/MS 1.5; MS/HE 0.5; HE/head length in frontal view 0.7; widths head/mesoscutum 1.2. Antenna. Pedicel + flagellum length/mesoscutum width 1.8; pedicel + flagellum length/head width 1.5 ; lengths scape/ventral plaque 2.2 ; ventral plaque located above the middle of scape; scape length/width 3.3; lengths scape/head (dorsal view) 0.6; scape length/HE 0.9; length/width F1, F2, F3, F4, clava: 2.3, 2.2, 2.0, 2.0, 5.0; length dorsobasal setae on F1/length F1 0.6. Mesosoma. Length/width 1.6; mesoscutum length/width 0.6; mesoscutellum length/width 0.8; widths SMG/ SLG 1.2; enclosed space between SMG length/width 2.2; lengths mesoscutum/mesoscutellum 1.3; lengths mesoscutellum/dorsellum 3.0; lengths mesosoma/gaster 0.8. Wings. CC length/width 30.0; lengths CC/MV 1.0; lengths MV/ST 1.9; lengths MV/ PM 5.8; lengths PM/ST 0.3; submarginal vein with eight setae on dorsal surface, in both types. Gaster. With lateral tufts of pale and flattened setae on $\mathrm{Gt}_{5-6}$.

\section{Eriastichus scalaris sp. nov.}

http://zoobank.org/F4846532-08CB-4CE2-AB52-5FCE0689B574

Figure 52

Type locality. Costa Rica, San José, San Gerardo de Dota, 9³3'N, 8347'W, 20-21. ii.2013, J.S. Noyes leg.

Type specimen. Holotype male dried and glued to a paper card. Original labels: "COSTA RICA, San José, San Gerardo de Dota, 20-21.ii.2013, J.S. Noyes, NHM (Ent) 2012-91", "HOLOTYPE Eriastichus scalaris Hansson" [red printed label], (NHMUK014431067).

Diagnosis (male). Head dark brown with part below antennaltoruli pale brown, scape pale brown, pedicel and flagellum brown; ventral plaque on scape ca. $0.4 \times$ as long as scape (Fig. 52), antenna with dorsobasal setae on F1 $1.0 \times$ as long as F1; gaster with lateral tufts of pale and flattened setae on $\mathrm{Gt}_{5-6}$. 
Description (male holotype NHMUK014431067). Length of body $1.5 \mathrm{~mm}$. Head dark brown with part below antennaltoruli pale brown, scape pale brown, pedicel and flagellum brown, ventral plaque dark brown. Mesoscutum, mesoscutellum and propodeum dark brown, dorsellum pale brown. Legs with fore and mid coxae pale brown, hind coxa and hind femur dark brown, remaining parts of legs yellowish brown. Gaster dark brown.

Head. Length/width in frontal view 0.8; width/length in dorsal view 2.1; POL/ OOL 2.0; WM/MS 1.4; MS/HE 0.7; HE/head length in frontal view 0.5; widths head/mesoscutum 1.1. Antenna. Pedicel + flagellum length/mesoscutum width 2.0; pedicel + flagellum length/head width 1.7; lengths scape/ventral plaque 2.5; ventral plaque located in the middle of scape; scape length/width 3.1; lengths scape/head (dorsal view) 0.5; scape length/HE 1.0; length/width F1, F2, F3, F4, clava: 2.5, 2.3, 2.5, 2.5, 5.8; length dorsobasal setae on F1/length F1 1.0. Mesosoma. Length/width 1.6; mesoscutum length/width 0.7; mesoscutellum length/width 0.5; widths SMG/SLG 0.9; enclosed space between SMG length/width 2.4; lengths mesoscutum/mesoscutellum 1.5; lengths mesoscutellum/dorsellum 3.0; lengths mesosoma/gaster 0.8. Wings. CC length/width 16.0; lengths CC/MV 1.0; lengths MV/ST 2.2; lengths MV/PM 7.1; lengths PM/ST 0.3; submarginal vein with seven setae on dorsal surface. Gaster. With lateral tufts of pale and flattened setae on $\mathrm{Gt}_{5-6}$.

\section{Eriastichus sodalis sp. nov.}

http://zoobank.org/F0A17B16-B048-4891-AF6C-3184ED9B3A21

Figure 48

Type locality. Costa Rica, San José, San Gerardo de Dota, 9³3'N, 8347'W, 20-21. ii.2013, J.S. Noyes leg.

Type specimen. Holotype male dried and glued to a paper card. Original labels: "COSTA RICA, San José, San Gerardo de Dota, 20-21.ii.2013, J.S. Noyes, NHM (Ent) 2012-91", "HOLOTYPE Eriastichus sodalis Hansson" [red printed label], (NHMUK014431068).

Diagnosis (male). Head dark brown with metallic tinges, scape pale brown, pedicel and flagellum dark brown; ventral plaque on scape ca. $0.3 \times$ as long as scape (Fig. 48), antenna with dorsobasal setae on F1 $0.8 \times$ as long as F1; gaster with lateral tufts of pale and flattened setae on $\mathrm{Gt}_{6}$.

Description (male holotype NHMUK014431068). Length of body $1.9 \mathrm{~mm}$. Head dark brown with metallic tinges, scape pale brown, pedicel and flagellum dark brown, ventral plaque dark brown. Mesoscutum and mesoscutellum black with metallic tinges, dorsellum dark brown, propodeum black. Legs with mid coxa and hind femur pale brown, hind coxa dark brown, remaining parts of legs yellowish brown. Gaster dark brown.

Head. Length/width in frontal view 0.8; width/length in dorsal view 2.2; POL/ OOL 1.6; WM/MS 1.6; MS/HE 0.5; HE/head length in frontal view 0.6; widths 
head/mesoscutum 1.1. Antenna. Pedicel + flagellum length/mesoscutum width 2.0; pedicel + flagellum length/head width 1.9; lengths scape/ventral plaque 3.0; ventral plaque located in the middle of scape; scape length/width 2.9; lengths scape/head (dorsal view) 0.5; scape length/HE 0.9; length/width F1, F2, F3, F4, clava: 2.9, 2.6, 2.6, 2.4, 5.7; length dorsobasal setae on F1/length F1 0.8. Mesosoma. Length/width 1.7; mesoscutum length/width 0.7; mesoscutellum length/width 0.8; widths SMG/SLG 1.1; enclosed space between SMG length/width 2.2; lengths mesoscutum/mesoscutellum 1.5; lengths mesoscutellum/dorsellum 2.8; lengths mesosoma/gaster 0.7. Wings. CC length/width 19.3; lengths CC/MV 1.0; lengths MV/ST 2.2; lengths MV/PM 5.3; lengths PM/ST 0.4; submarginal vein with six setae on dorsal surface. Gaster. With lateral tufts of pale and flattened setae on $\mathrm{Gt}_{6}$.

\section{Eriastichus taraxis sp. nov.}

http://zoobank.org/F6E6E4E6-E788-4C49-BA77-2965F2B87E70

Figure 53

Type locality. Costa Rica, Puntarenas, Estación Biológica Monteverde, $10^{\circ} 20^{\prime} \mathrm{N}$, $84^{\circ} 49^{\prime} \mathrm{W}, 1540 \mathrm{~m}, 26 . i i .2007$, J.S. Noyes leg.

Type specimen. Holotype male dried and glued to a paper card. Original labels: "COSTA RICA, Puntarenas, E.B. Monteverde, $10^{\circ} 20^{\prime} \mathrm{N}, 84^{\circ} 49^{\prime} \mathrm{W}, 1540 \mathrm{~m}$, 26.ii.2007, J.S. Noyes", "HOLOTYPE Eriastichus taraxis Hansson" [red printed label], (NHMUK014431069).

Diagnosis (male). Head dark brown, scape yellowish brown, pedicel pale brown, flagellum brown; ventral plaque on scape ca. $0.4 \times$ as long as scape (Fig. 53), antenna with dorsobasal setae on F1 $0.8 \times$ as long as F1; gaster with lateral tufts of pale and flattened setae on $\mathrm{Gt}_{6}$.

Description (male holotype NHMUK014431069). Length of body $1.6 \mathrm{~mm}$. Head dark brown, scape yellowish brown, pedicel pale brown, flagellum brown, ventral plaque dark brown. Mesoscutum and mesoscutellum black with metallic tinges, dorsellum and propodeum dark brown. Legs yellowish brown, hind coxa with base dark brown. Gaster dark brown.

Head. Length/width in frontal view 0.8 ; width/length in dorsal view 2.1; POL/ OOL 2.1; WM/MS 1.5; MS/HE 0.5; HE/head length in frontal view 0.6; widths head/mesoscutum 1.1. Antenna. Pedicel + flagellum length/mesoscutum width 2.1; pedicel + flagellum length/head width 1.9; lengths scape/ventral plaque 2.7; ventral plaque located in the middle of scape; scape length/width 3.1; lengths scape/head (dorsal view) 0.5; scape length/HE 0.8; length/width F1, F2, F3, F4, clava: 3.0, 3.0, 3.0, 2.8, 6.3; length dorsobasal setae on F1/length F1 0.8. Mesosoma. Length/width 1.6; mesoscutum length/width 0.7 ; mesoscutellum length/width 0.8 ; widths SMG/SLG 1.1 ; enclosed space between SMG length/width 2.3; lengths mesoscutum/mesoscutellum 1.6; lengths mesoscutellum/dorsellum 2.8; lengths mesosoma/gaster 0.7. Wings. CC length/width 22.5; lengths CC/MV 1.0; lengths MV/ST 2.0; lengths MV/PM 
6.5; lengths PM/ST 0.3; submarginal vein with seven setae on dorsal surface. Gaster. With lateral tufts of pale and flattened setae on $\mathrm{Gt}_{6}$.

\section{Eriastichus tendrilis sp. nov.}

http://zoobank.org/1EF86DE6-2C33-48A8-9555-08C10059AC76

Figure 55

Type locality. Costa Rica, Puntarenas, Estación Biológica Monteverde, $10^{\circ} 20^{\prime} \mathrm{N}$, $84^{\circ} 49^{\prime} \mathrm{W}, 1540 \mathrm{~m}, 18-25 . i i .2004$, C. Hansson leg.

Type specimen. Holotype male dried and glued to a paper card. Original labels: "COSTA RICA, Puntarenas, E.B Monteverde, $10^{\circ} 20^{\prime} \mathrm{N}, 84^{\circ} 49^{\prime} \mathrm{W}, 1540 \mathrm{~m}, 18-25$. ii.2004, C. Hansson", "HOLOTYPE Eriastichus tendrilis Hansson" [red printed label], (MZLU:7048.1).

Additional type material. Paratypes $(5 \hat{\jmath})$ ): $2 \hat{\jmath} \hat{o}$ with same label data as holotype (MZLU:7048.2, MZUCR:01696); $2 \widehat{ } \widehat{0}$ from same locality as holotype but collected 26.ii.2007 (NHMUK014431070, NHMUK014431071); 10 ‘̄ "COSTA RICA, San José, San Gerardo de Dota, El Manantial, 18-20.ii.2010, J.S. Noyes, NHM (Ent) 2010-21" (NHMUK014431072).

Diagnosis (male). Head black with metallic tinges, scape yellowish brown, pedicel and flagellum brown; ventral plaque on scape ca. $0.3 \times$ as long as scape (Fig. 55), antenna with dorsobasal setae on F1 $0.6 \times$ as long as F1; gaster with lateral tufts of pale and flattened setae on $\mathrm{Gt}_{6}$.

Description (male holotype MZLU:7048.1). Length of body $1.5 \mathrm{~mm}$ (paratypes 1.4-1.7 mm). Head black with metallic tinges, scape yellowish brown, pedicel and flagellum brown, ventral plaque dark brown. Mesoscutum and mesoscutellum black with metallic tinges, dorsellum and propodeum black. Legs with fore and mid coxae yellowish brown with base brown, hind coxa black, hind femur pale brown, remaining parts of legs yellowish brown. Gaster dark brown.

Head. Length/width in frontal view 0.7 ; width/length in dorsal view 2.5; POL/ OOL 1.8; WM/MS 1.6; MS/HE 0.6; HE/head length in frontal view 0.6; widths head/mesoscutum 1.1. Antenna. Pedicel + flagellum length/mesoscutum width 1.5; pedicel + flagellum length/head width 1.5; lengths scape/ventral plaque 3.0; ventral plaque located in the middle of scape; scape length/width 3.0; lengths scape/head (dorsal view) 0.6; scape length/HE 0.9; length/width F1, F2, F3, F4, clava: 2.7, 2.7, 2.5, 2.5, 5.5; length dorsobasal setae on F1/length F1 0.6. Mesosoma. Length/ width 1.5; mesoscutum length/width 0.7 ; mesoscutellum length/width 0.8 ; widths SMG/SLG 1.0; enclosed space between SMG length/width 2.0; lengths mesoscutum/mesoscutellum 1.6; lengths mesoscutellum/dorsellum 3.0; lengths mesosoma/ gaster 1.0. Wings. CC length/width 18.0; lengths CC/MV 1.0; lengths MV/ST 2.1; lengths MV/PM 6.5; lengths PM/ST 0.3; submarginal vein with six setae on dorsal surface (7-8 setae in paratypes). Gaster. With lateral tufts of pale and flattened setae on $\mathrm{Gt}_{6}$. 


\section{Eriastichus tonioazofeifai sp. nov.}

http://zoobank.org/4E666BE9-F215-4776-AA27-1E865C9F7A12

Figure 57

Type locality. Costa Rica, Heredia, Estación Biológica La Selva, 100-200 m, 10²6'N, 8401'W, 30-31.iii.2002, swept, J.A. Azofeifa leg.

Type specimen. Holotype male dried and glued to a paper card. Original labels: "COSTA RICA, Heredia, E.B. La Selva, 100-200 m, 10²6'N, 8401'W, 30-31. iii.2002, swept, J.A. Azofeifa,", "HOLOTYPE Eriastichus tonioazofeifai Hansson" [red printed label], (MZLU:7049.1).

Diagnosis (male). Head dark brown with part below antennal toruli yellowish brown; antenna (Fig. 57): scape pale brown, ventral plaque on scape ca. $0.1 \times$ as long as scape, antenna with dorsobasal setae on F1 $1.1 \times$ as long as F1; gaster with lateral tufts of pale and flattened setae on $\mathrm{Gt}_{6}$.

Description (male holotype MZLU:7049.1). Length of body $1.3 \mathrm{~mm}$. Head dark brown with part below antennal toruli yellowish brown. Antenna with scape pale brown, ventral plaque dark brown, pedicel and flagellum brown. Mesoscutum and mesoscutellum dark brown with metallic tinges; dorsellum pale brown; propodeum dark brown. Legs yellowish brown with hind coxa and hind femur pale brown. Wings \pm hyaline. Gaster dark brown.

Head. Length/width in frontal view 0.7 ; width/length in dorsal view 2.3; POL/ OOL 2.5; WM/MS 1.5; MS/HE 0.5; HE/head length in frontal view 0.6; widths head/mesoscutum 1.2. Antenna. Pedicel + flagellum length/mesoscutum width 2.0; pedicel + flagellum length/head width 1.6; lengths scape/ventral plaque 8.0; ventral plaque located in the middle of scape; scape length/width 5.8; lengths scape/head (dorsal view) 0.8; scape length/HE 1.3; length/width F1, F2, F3, F4, clava: 2.0, 2.0, 2.0, 2.2, 5.3; length dorsobasal setae on F1/length F1 1.1. Mesosoma. Length/width 1.5; mesoscutum length/width 0.6; mesoscutellum length/width 0.7; widths SMG/SLG 1.1; enclosed space between SMG length/width 2.0; lengths mesoscutum/mesoscutellum 1.4; lengths mesoscutellum/dorsellum 2.9; lengths mesosoma/gaster 0.7. Wings. CC length/width 21.0; lengths CC/MV 1.3; lengths MV/ST 1.9; lengths MV/PM 3.6; lengths PM/ST 0.5; submarginal vein with six setae on dorsal surface. Gaster. With lateral tufts of pale and flattened setae on $\mathrm{Gt}_{6}$.

Etymology. Named after collector, Antonio (Tonio) Azofeifa, parataxonomist in Costa Rica.

\section{Eriastichus velaminis sp. nov.}

http://zoobank.org/1263C4A3-410D-4C60-A610-8F8CF591E7A9

Figure 56

Type locality. Costa Rica, San José, San Gerardo de Dota, 9³3'N, 8347'W, 20-21. ii.2013, J.S. Noyes leg. 
Type specimen. Holotype male dried and glued to a paper card. Original labels: "COSTA RICA, San José, San Gerardo de Dota, 20-21.ii.2013, J.S. Noyes, NHM (Ent) 2012-91", "HOLOTYPE Eriastichus velaminis Hansson" [red printed label], (NHMUK014431073).

Diagnosis (male). Head dark brown with metallic tinges, scape and pedicel pale brown, flagellum brown; ventral plaque on scape ca. $0.4 \times$ as long as scape (Fig. 56), antenna with dorsobasal setae on F1 $0.8 \times$ as long as F1; gaster with lateral tufts of pale and flattened setae on $\mathrm{Gt}_{6}$.

Description (male holotype NHMUK014431073). Length of body $1.2 \mathrm{~mm}$. Head dark brown with metallic tinges, scape and pedicel pale brown, flagellum brown, ventral plaque dark brown. Mesoscutum and mesoscutellum black with metallic tinges, dorsellum and propodeum dark brown. Legs with coxae and femora on fore and mid legs pale brown, hind coxa and hind femur dark brown, remaining parts of legs yellowish brown. Gaster dark brown.

Head. Length/width in frontal view 0.8 ; width/length in dorsal view 2.1; POL/ OOL 1.9; WM/MS 1.4; MS/HE 0.6; HE/head length in frontal view 0.5; widths head/mesoscutum 1.1. Antenna. Pedicel + flagellum length/mesoscutum width 1.8; pedicel + flagellum length/head width 1.5; lengths scape/ventral plaque 2.8; ventral plaque located below the middle of scape; scape length/width 3.1; lengths scape/head (dorsal view) 0.5; scape length/HE 1.0; length/width F1, F2, F3, F4, clava: 2.4, 2.4, 2.2, 2.0, 5.4; length dorsobasal setae on F1/length F1 0.8. Mesosoma. Length/width 1.5; mesoscutum length/width 0.6; mesoscutellum length/width 0.8; widths SMG/ SLG 1.1; enclosed space between SMG length/width 2.0; lengths mesoscutum/mesoscutellum 1.5; lengths mesoscutellum/dorsellum 2.5; lengths mesosoma/gaster 0.8. Wings. CC length/width 22.0; lengths CC/MV 1.0; lengths MV/ST 2.1; lengths MV/PM 4.7; lengths PM/ST 0.5; submarginal vein with five setae on dorsal surface. Gaster. With lateral tufts of pale and flattened setae on $\mathrm{Gt}_{6}$.

\section{Eriastichus vestis sp. nov.}

http://zoobank.org/59E0A806-AAE9-4C16-B373-23BBE4FE3BDC Figure 58

Type locality. Costa Rica, Puntarenas, San Vito, Estación Biológica Las Alturas, $1500 \mathrm{~m}, 8^{\circ} 57^{\prime} \mathrm{N}, 82^{\circ} 50^{\prime} \mathrm{W}, 17-18 . i i .2012$, J.S. Noyes leg.

Type specimen. Holotype male dried and glued to a paper card. Original labels: "COSTA RICA, Puntarenas, San Vito, E.B. Las Alturas, 1500 m, 8 $57^{\prime} \mathrm{N}, 82^{\circ} 50^{\prime} \mathrm{W}$, 17-18.ii.2012, J.S. Noyes, NHM (Ent) 2012-91", "HOLOTYPE Eriastichus vestis Hansson" [red printed label], (NHMUK014431074).

Diagnosis (male). Head dark brown, scape and pedicel pale brown, flagellum brown; ventral plaque on scape ca. $0.3 \times$ as long as scape (Fig. 58), antenna with dorsobasal setae on F1 $0.9 \times$ as long as F1; gaster with lateral tufts of pale and flattened setae on $\mathrm{Gt}_{6}$. 


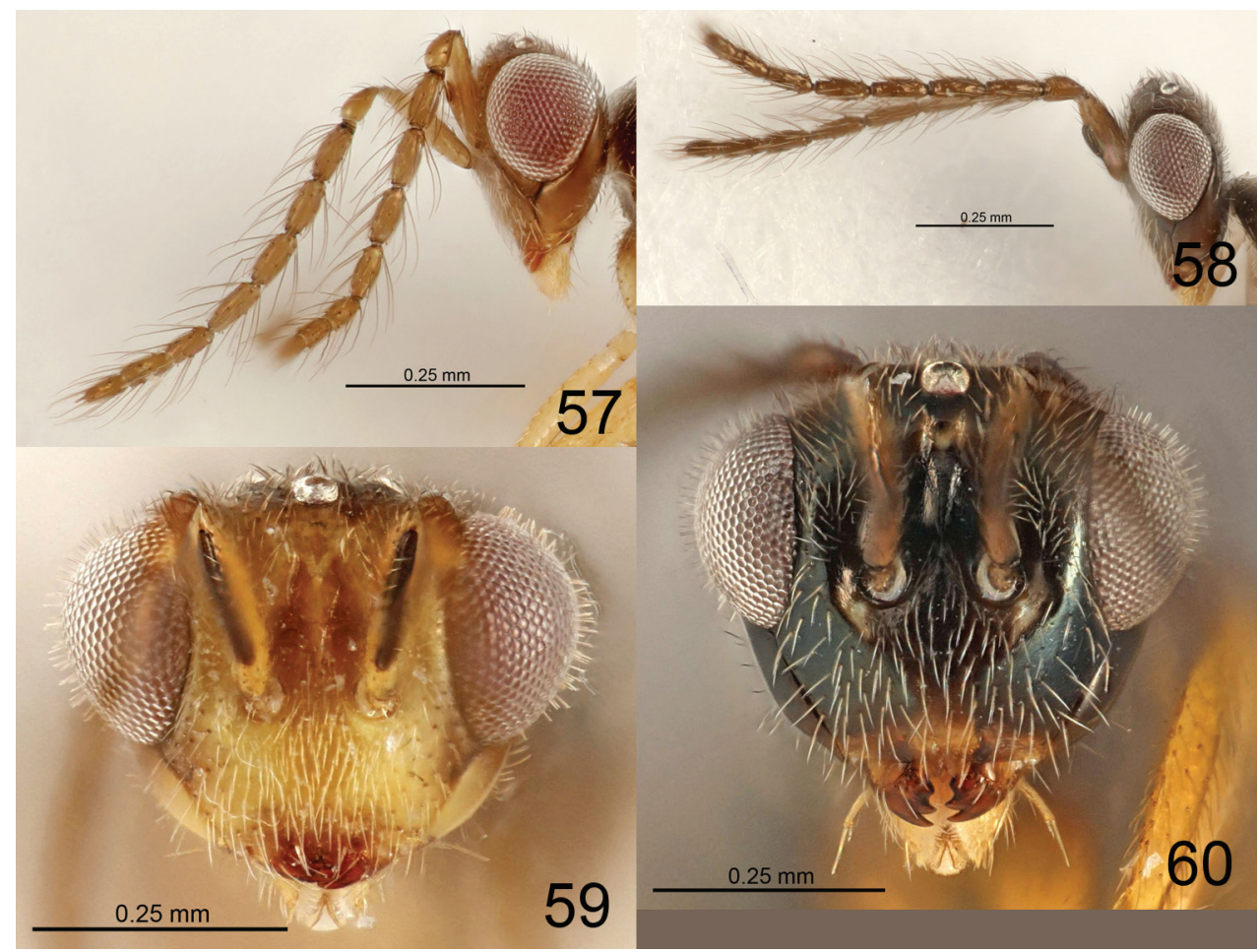

Figures 57-60. Eriastichus spp. 57, 58 head and antenna in lateral view, male holotypes: $\mathbf{5 7}$ E. tonioazofeifai $\mathbf{5 8}$ E. vestis; $\mathbf{5 9 ,} \mathbf{6 0}$ head in frontal view, male holotypes: $\mathbf{5 9}$ E. pallidops $\mathbf{6 0}$ E. denotatis.

Description (male holotype NHMUK014431074). Length of body $1.5 \mathrm{~mm}$. Head dark brown, scape and pedicel pale brown, flagellum brown, ventral plaque dark brown. Mesoscutum, mesoscutellum and propodeum black with metallic tinges, dorsellum dark brown. Legs with coxae and femora dark brown, remaining parts of legs yellowish brown. Gaster dark brown.

Head. Length/width in frontal view 0.8 ; width/length in dorsal view 2.4; POL/ OOL 1.9; WM/MS 1.5; MS/HE 0.6; HE/head length in frontal view 0.5; widths head/mesoscutum 1.0. Antenna. Pedicel + flagellum length/mesoscutum width 1.8; pedicel + flagellum length/head width 1.8; lengths scape/ventral plaque 2.9; ventral plaque located above the middle of scape; scape length/width 2.9; lengths scape/head (dorsal view) 0.5; scape length/HE 1.0; length/width F1, F2, F3, F4, clava: 3.0, 3.2, 3.0, 3.0, 7.4; length dorsobasal setae on F1/length F1 0.9. Mesosoma. Length/width 1.5; mesoscutum length/width 0.6; mesoscutellum length/width 0.7; widths SMG/ SLG 1.5; enclosed space between SMG length/width 1.7; lengths mesoscutum/mesoscutellum 1.4; lengths mesoscutellum/dorsellum 2.5; lengths mesosoma/gaster 0.9. Wings. CC length/width 16.0; lengths CC/MV 1.0; lengths MV/ST 2.0; lengths MV/PM 5.3; lengths PM/ST 0.4; submarginal vein with five setae on dorsal surface. Gaster. With lateral tufts of pale and flattened setae on $\mathrm{Gt}_{6}$. 


\section{Discussion}

The original description of Eriastichus was based on relatively few specimens and was a morphologically distinct genus. With the addition here of some unique features on the gaster of both sexes, the inflated pleural membrane between $\mathrm{Gt}_{1-4}$ and $\mathrm{Gs}_{1-4}$, and the tuft of pale and flattened setae laterally on $\mathrm{Gt}_{4-6,}$ as well as the features given by $\mathrm{La}$ Salle in the original description, Eriastichus forms a morphologically well-defined and quite remarkable genus of the Tetrastichinae.

Species included here can only be separated through morphological characters in males, mainly on the antennae, as demonstrated in the identification key. Apart from the antennae, males of the different species are very similar, with the exception of variation in colour on the head and legs in some of the species. The antennae in females show very little variation, which is also the case for other morphological features. Thus, females cannot be separated to species, and they cannot be linked to conspecific males using morphology. Molecular data will be essential for the association of sexes of conspecific specimens, and for the identification of females.

The three species included in the original description were based mainly on females, and with relatively few specimens. Two of the species had the mesoscutellum with parts lateral to submedian grooves strongly setose, a character state not present in any of the new species described here. The third species included males from Mexico, separated in the key from species described here.

Most of the newly described species are represented by single specimens, thus eliminating the possibility of describing morphological variation within species. However, in species with two or more specimens, frequently from different localities and different collecting events, the antennal characters used to separate species show very little or no variation.

\section{Acknowledgements}

I am very grateful to John S. Noyes (NHMUK) for loan of material, and to Paul E. Hanson (University of Costa Rica) for valuable comments on the manuscript. The Microscopy Facility at the Department of Biology, Lund University, is acknowledged for the use of their scanning electron microscope.

\section{References}

Gibson GAP (1997) Morphology and terminology. In: Gibson GAP, Huber JT, Woolley JB (Eds) Annotated keys to the genera of Nearctic Chalcidoidea (Hymenoptera). NRC Research Press, Ottawa, Canada, 16-44. https://doi.org/10.1093/aesa/91.3.359

Hansson C (2020) Lasalleistichus a new genus of Tetrastichinae (Hymenoptera: Eulophidae) from the Neotropical region, including four new species. Journal of Natural History 54: 621-633. https://doi.org/10.1080/00222933.2020.1715500 
International Commission of Zoological Nomenclature (1999) International code of zoological nomenclature : Code international de nomenclature zoologique $\left(4^{\text {th }} \mathrm{ed}\right.$.). International Trust for Zoological Nomenclature, c/o Natural History Museum, London.

La Salle J (1994) North American genera of Tetrastichinae (Hymenoptera: Eulophidae). Journal of Natural History 28: 109-236. https://doi.org/10.1080/00222939400770091

La Salle J, Schauff ME, Hansson C (2006) Familia Eulophidae. In: Hanson PE, Gauld ID (Eds) Hymenoptera de la Región Neotropical. The American Entomological Institute, Gainesville, Florida, USA, 356-374. [in Spanish] 